\title{
Flora arbustivo-arbórea dos planaltos da Bodoquena e de Maracaju, na porção centro-sudoeste do estado do Mato Grosso do Sul, Brasil
}

\author{
Vivian Ribeiro Baptista Maria', Fabrício de Souza Maria' \& Sandro Menezes Silva ${ }^{2}$
}

\begin{abstract}
' Bíon Consultoria e Assessoria Ambiental. Av. Pilád Rebuá, n.347, Centro, CEP 79.290-000, Bonito, MS, Brasil. vivian@bionconsultoria.com
2 Universidade Federal da Grande Dourados, Faculdade de Ciências Biológicas e Ambientais, Rodovia Dourados-Itahum, km 12 ، Caixa Postal 322, CEP 79.804-970, Dourados, MS, Brasil.
\end{abstract}

\begin{abstract}
RESUMO - O Mato Grosso do Sul situa-se em zona transicional entre a Mata Atlântica, o Cerrado, o Pantanal e o Chaco; predominam formações florestais e savânicas, com florística resultante da mistura de elementos destes biomas. Apesar da sua relevância biogeográfica, são poucos estudos florísticos no estado. É apresentado um checklist das espécies arbustivo-arbóreas da porção centro-sudoeste da Bacia do Alto Paraguai, resultado de coletas e observações de campo e da compilação de trabalhos publicados. São 507 espécies, 64 famílias e 258 gêneros. Fabaceae (102), Myrtaceae (30), Malvaceae (21), Euphorbiaceae (17), Bignoniaceae (16), Annonaceae e Rubiaceae (15 espécies cada) são as famílias mais ricas. Duas espécies ocorreram em todos os sítios enquanto 103 espécies ocorreram somente em uma área. Houve contribuição diferenciada na composição florística dos biomas vizinhos, sendo Mata Atlântica e Cerrado os que mais influenciaram. Treze espécies integram a Lista Vermelha de Espécies Ameaçadas da International Union for Conservation of Nature - IUCN enquanto três estão na Lista Brasileira da Flora Ameaçada de Extinção.
\end{abstract}

Palavras-chave: Cerrado, Chaco, Fabaceae, fitodiversidade, Floresta Estacional

ABSTRACT - Wood flora of Bodoquena and Maracaju Plateau, middle-southeastern region of Mato Grosso do Sul state, Brazil. Mato Grosso do Sul state is a transitional zone among four biomes: Atlantic Forest, Cerrado, Chaco and Pantanal. Forest and savanna are the main vegetation types and floristic composition results from the combination of elements of these biomes depending on the specific environmental factors. There are few published studies on the floristic structure and its diversified environmental situations. We present a checklist of woody species from the Upper Paraguay Basin as a result of field observations and inventories besides a compilation of published data. 507 species, 64 families and 258 genera were listed. Fabaceae (102), Myrtaceae (30), Malvaceae (21), Euphorbiaceae (17), Bignoniaceae (16), Annonaceae and Rubiaceae (15 species each) were the richest families. Two species occurred at all surveyed sites while 103 species occurred only in one site. Atlantic Forest and Cerrado were the biomes with greatest influence. Thirteen species are on the IUCN Red List of Threatened Species while three are on the Brazilian Flora Endangered Species List.

Keywords: Chaco, Fabaceae, plant diversity, Savanna e Seasonal Forest

\section{INTRODUÇÃO}

O estado de Mato Grosso do Sul localiza-se na região Centro-Oeste do Brasil, limitando-se ao norte com o Mato Grosso, a nordeste com Goiás e Minas Gerais, a leste com São Paulo, a sudeste com o Paraná, ao sul e parte do sudeste com o Paraguai e a oeste com a Bolívia. Tem área aproximada de $358.000 \mathrm{Km}^{2}$, o que equivale a $4,2 \%$ do território brasileiro. $\mathrm{O}$ estado está situado em uma grande região de transição em termos biogeográficos, com áreas representativas de Savana (Cerrado e Chaco) e Florestas Estacionais, com diversas variações estruturais nos diferentes pontos do estado (Brasil 1997, Silva et al. 2011).

As Savanas, no Brasil denominadas de Cerrado, estão representadas por diversas fitofisionomias, desde campos limpos e abertos até formações florestais conhecidas popularmente como Cerradão. Já as Florestas são representadas por extensões mais interiorizadas das florestas úmidas da região leste do Brasil, relacionadas ao bioma Mata Atlântica, e por áreas representativas do oeste do continente, especialmente da região leste da Bolívia, conhecida como "Bosque Seco Chiquitano" (VidesAlmonacid et al. 2007), que abriga florestas decíduas e áreas de cerrado, formando um mosaico de tipos de vegetação florestal. Em termos de relevo, a região oeste do Mato Grosso do Sul caracteriza-se, em sua maior extensão, pela planície que forma o Pantanal, circundada por áreas mais elevadas representativas das bordas do Planalto Central Brasileiro, conjunto que na região é conhecido como Planalto de Maracaju, e na porção sudoeste, pelo Planalto da Bodoquena, que representa a borda de um planalto erodido formado por rochas calcárias antigas e feições associadas. Morros isolados desses dois conjuntos ocorrem em alguns pontos mais a oeste, na divisa com a Bolívia e o Paraguai, e representam as últimas elevações ao leste desses países antes de entrar no território brasileiro. Na região de Porto Murtinho, na divisa com o Paraguai, destaca-se a região denominada de Morraria do Amonquijá, onde se situa a parte final da planície do Pantanal, marcada por terras altas denominadas localmente de "Fecho dos Morros".

O presente artigo traz um checklist das espécies arbustivo-arbóreas das partes altas da Bacia do Alto 
Paraguai, na sua porção centro-sudoeste. A região incluída nesse levantamento, conforme Brasil (1997) é denominada de "Terras altas na região Sul", que inclui o "Planalto de Maracaju-Campo Grande", o "Planalto da BodoquenaBonito", o "Planalto do Amonquijá" e a "Depressão do Miranda". Resulta de diversos esforços de coleta e observação dos autores nessas regiões e de uma compilação de dados publicados em periódicos nacionais, incluindo somente as espécies arbustivas e arbóreas registradas nesses levantamentos.

As principais lacunas do conhecimento sobre a flora arbustiva-arbórea relacionam-se às áreas que ainda precisam ser melhor investigadas em campo, com levantamentos quali-quantitativos da flora e da vegetação, pois como a região é uma grande área de transição entre importantes biomas da América do Sul, pode apresentar diversas novidades taxonômicas

\section{MATERIAL E MÉTODOS}

A listagem de espécies apresentada resultou da compilação de levantamentos florísticos realizados em 28 sítios amostrais da região centro-sudoeste do Mato Grosso do Sul, mais especificamente em porções dos planaltos da Bodoquena e de Maracaju, nos municípios de Porto Murtinho (seis sítios amostrais), Bonito (cinco), Jardim (cinco), Aquidauana (cinco), Bodoquena (três), Corguinho (dois), Rio Negro (um) e Miranda (um) (Quadro 1) Estas áreas foram estudadas com a finalidade de realização de zoneamento para estabelecimento de um projeto de restauração ambiental (Bacia do Córrego do Café); diagnóstico do meio biótico (flora) para caracterização do Corredor de Biodiversidade Miranda-Serra da Bodoquena (Fases II e IV); diagnóstico da vegetação para proposição de corredores ecológicos entre propriedades rurais arrendadas para produção florestal (eucalipto) "Projeto Reflorestar Legal" e elaboração dos planos de manejos de reservas privadas - Reserva Particular do Patrimônio Natural RPPN. Além das informações dessas áreas, foram incluídas ainda as listagens publicadas em periódicos científicos, obtidas por meio de pesquisa em bases eletrônicas de literatura: Battilani et al. (2005), Bueno et al. (2007) e Baptista-Maria et al. (2009).

A nomenclatura empregada aos tipos vegetacionais ocorrentes nas áreas estudadas seguiu a proposta de classificação de Silva et al. (2011), com uma modificação especificamente para as florestas situadas ao longo dos cursos de água, que seguiu o proposto por Rodrigues (2004). Com relação às tipologias vegetacionais "Floresta Estacional Perenifólia" e "Floresta Paludosa" utilizou-se proposições feitas por Kunz et al. (2009) e Teixeira \& Assis (2005), respectivamente.

As coleções que resultaram nesse trabalho estão depositadas nos herbários da Universidade Federal do Mato Grosso do Sul e da Escola Superior de Agricultura Luiz de Queiroz, São Paulo.

O método utilizado na maioria das áreas, com exceção somente dos estudos de Battilani et al. (2005) e de Bueno et al. (2007), nos quais foi usado o método de amostragem por parcelas, foi o levantamento qualitativo por tempo de avaliação, sendo a amostragem por meio de caminhadas passando por toda a área florestal, até que não aparecessem novas espécies em 15 minutos de amostragem contínua. Procurou-se estabelecer o mesmo número de horas amostrais nas áreas estudadas, sendo coletadas as espécies em fase reprodutiva, com repetição de coletas de uma mesma espécie quando encontrada em diferentes fenofases. A coleta do material botânico de referência foi realizada pelos métodos usuais de herborização, sendo o tempo de coleta e preparação do material descontado do tempo total de amostragem. O material coletado foi determinado com auxilio de literatura especializada, comparações com exsicatas existentes em herbários e consulta a especialistas.

A relação de famílias, gêneros e espécies, com seus respectivos autores, foi verificada na base de dados da Lista de Espécies da Flora do Brasil (2012), Plant List (2012) e Trópicos (2012)..

\section{RESULTADOS E DISCUSSÃO}

O número total de espécies identificadas nas áreas amostrais foi de 507, distribuídas em 64 famílias e 258 gêneros (Quadro 2). As famílias botânicas mais representativas em número de espécies foram Fabaceae (102 espécies - 1/5 do total amostrado), seguida por Myrtaceae (30 espécies), Malvaceae (21 espécies), Euphorbiaceae (17 espécies), Bignoniaceae (16 espécies), Annonaceae e Rubiaceae (15 espécies cada). Estes resultados seguem padrões florísticos já registrados por Felfili et al. (2001) e Nunes et al. (2003), que citam as famílias Fabaceae e Myrtaceae como as mais representativas em número de espécies arbustivo-arbóreas nas florestas ribeirinhas do estado de Mato Grosso do Sul. Dentre as espécies, 103 (20,3\%) foram registradas em apenas uma das áreas amostradas e duas espécies Pterogyne nitens e Casearia sylvestris ocorreram em todas as áreas amostrais. Tapirira guianensis, Myracrodruon urundeuva, Cecropia pachystachya, Myrsine guianensis e Psychotria carthagenensis ocorreram em 14 das 15 áreas amostradas, seguidas por Handroanthus impetiginosus, Anadenanthera colubrina, Maclura tinctoria, Rhamnidium elaeocarpum, Trema micrantha, Guazuma ulmifolia e Piper aduncum que ocorreram em 13 sítios estudados. Nas áreas amostradas, as espécies arbóreas representaram 75,3\% (382 espécies) do total amostrado, os arbustos totalizaram 22,1\% (112 espécies) e as palmeiras somaram 2,6\% (13 espécies).

As florestas estacionais compõem no Brasil Central, um extenso "arquipélago" composto de ilhas ao longo de vales férteis e afloramentos de rochas de origem calcária (Felfili 2003). As florestas estacionais ribeirinhas formam corredores que funcionam como refúgios para espécies das florestas Atlântica, Amazônica e das florestas da Bacia do Paraná (Oliveira-Filho \& Ratter 1995) e contêm aproximadamente 2.031 espécies de fanerógamas (Felfili et al. 2001), representando 30\% da flora fanerogâmica do Brasil Central (Mendonça 
Quadro 1. Flora arbustivo-arbórea dos planaltos da Bodoquena e de Maracaju. Localidades incluídas nesse estudo com suas respectivas localizações e Fisonomia Florestal: FESR = Floresta Estacional Semidecidual Ribeirinha; FEDR = Floresta Estacional Decidual Ribeirinha; FESS $=$ Floresta Estacional Semidecidual Submontana; FEDS = Floresta Estacional Decidual Submontana; FEP = Floresta Estacional Perenifólia; FP = Floresta Paludosa; $\mathrm{SF}=$ Savana Florestada (Cerradão); SA = Savana Arborizada (Cerrado Senso Stricto); FPB = Formações Pioneiras (Buritizais); $\mathrm{SGL}=$ Savana Gramíneo - Lenhosa; SEP $=$ Savana Estépica Parque (Carandazais); SEA $=$ Savana Estépica Arborizada. ${ }^{1}=$ dados dos autores resultantes dos referidos trabalhos de consultoria.

\begin{tabular}{|c|c|c|c|c|}
\hline Local & Município & $\begin{array}{l}\text { Coordenada } \\
\text { Geográfica } \\
\text { (UTM SAD 69) }\end{array}$ & Fisionomia florestal & Fonte \\
\hline Faz. Baía Bonita & Bonito & $558834 / 7661138$ & FESR & \multirow{6}{*}{ Baptista-Maria et al. } \\
\hline Faz. América & Bonito & 546695 / 7651953 & FESR & \\
\hline Faz. Harmonia & Porto Murtinho & 525944 / 7653844 & FEDR & \\
\hline Faz. Campo Verde & Porto Murtinho & $529387 / 7644615$ & FESR & \\
\hline Faz. Santa Laura & Bonito & $527757 / 7703644$ & FESR & \\
\hline Faz. Rancho Branco & Bodoquena & $524303 / 7714717$ & FESR & \\
\hline Santuário do Prata & Jardim & $565645 / 7633441$ & FESR & Battilani et al. \\
\hline RPPN São Geraldo & Bonito & $545648 / 7648636$ & FESR & Bueno et al. \\
\hline Bacia do Córrego do Café & Rio Negro & $717007 / 7851759$ & SF, SA, FPB, FESR e FESS & $\begin{array}{c}\text { Adequação A. de } \\
\text { propriedades rurais do C. } \\
\text { do Café }{ }^{1}\end{array}$ \\
\hline RPPN Cabeceira do Prata & Jardim & $0559126 / 7629517$ & FESR e FESS, SF e SA & $\begin{array}{c}\text { Plano de Manejo da RPPN } \\
\text { Cabeceira do Prata }{ }^{1}\end{array}$ \\
\hline Córrego Agachi & Miranda & $0579635 / 7759565$ & FESR, FESS, SF, SA e SGL & \multirow{5}{*}{$\begin{array}{l}\text { Corredor de Biodiversidade } \\
\text { Miranda-SB (fase II) }{ }^{1}\end{array}$} \\
\hline Rio Salobra & Bodoquena & $0533688 / 7747785$ & FESR, FEDS, FESS e SA & \\
\hline Morraria do Fecho dos Morros & Porto Murtinho & $0404395 / 7625288$ & FEDS, SEP, FEDR, FESS, FESR & \\
\hline Morraria do Amonguijá & Porto Murtinho & $446320 / 7550600$ & FESS, FEDS, SEA e SGL & \\
\hline Rios Perdido e Apa: & Porto Murtinho & 0447154 / 7547863 & FESR e FEDS & \\
\hline RPPN Buraco das Araras & Jardim & 562155 / 7624231 & SF e SA & $\begin{array}{c}\text { Plano de Manejo da RPPN } \\
\text { Buraco das Araras }\end{array}$ \\
\hline Lagoa misteriosa & Jardim & $556988 / 7627940$ & FEDS e SF & $\begin{array}{c}\text { Plano de Manejo } \\
\text { Espeleológico da Lagoa } \\
\text { Misteriosa }^{1}\end{array}$ \\
\hline RPPN Fazenda da Barra & Bonito & $580200 / 7665660$ & FESR e SF & $\begin{array}{c}\text { Plano de Manejo da RPPN } \\
\text { Fazenda da Barra }\end{array}$ \\
\hline Faz. Taruana & Aquidauna & $683395 / 7727192$ & FESR, SA e SF & \multirow{5}{*}{ Projeto Reflorestar Legal ${ }^{1}$} \\
\hline Faz. Correntes & Aquidauna & 667114 / 7727957 & FESR, SA, SF e FEP & \\
\hline Faz. Vô Fiorindo & Aquidauna & $663981 / 7720971$ & SA, SF, FESR e FEP & \\
\hline Faz. Jatiúca & Aquidauna & $6209001 / 772947$ & SA, SF, FP e FEP & \\
\hline Faz. Boa Esperança & Aquidauna & $602367 / 7708141$ & SA, SF, FESR e FEP & \\
\hline Fecho dos Morros & Porto Murtinho & $0404395 / 7625288$ & FEDR, FEDS e SEP & $\begin{array}{l}\text { Corredor de Biodiversidade } \\
\text { Miranda-SB (fase IV) }\end{array}$ \\
\hline RPPN Cara da Onça & Bodoquena & $527321 / 7706420$ & FESR e FEDS & $\begin{array}{c}\text { Plano de Manejo da RPPN } \\
\text { Cara da Onça }{ }^{1}\end{array}$ \\
\hline RPPN Gavião de Penacho & Corguinho & $702100 / 7795028$ & SF e FESR & $\begin{array}{l}\text { Plano de Manejo da RPPN } \\
\text { Gavião de Penacho }\end{array}$ \\
\hline RPPN Vale do Bugio & Corguinho & 701753 / 7793533 & FESS, FESR, FEDS e SF & $\begin{array}{c}\text { Plano de Manejo da RPPN } \\
\text { Vale do Bugio }\end{array}$ \\
\hline RPPN Xodó do Vô Ruy & Jardim & 599528 / 7586371 & FEDS e SF & $\begin{array}{c}\text { Plano de Manejo da RPPN } \\
\text { Xodó do Vô Ruy }\end{array}$ \\
\hline
\end{tabular}


Quadro 2. Lista das espécies arbustivo-arbóreas dos planaltos da Bodoquena e de Maracaju, na porção centro-sudoeste do estado do Mato Grosso do Sul, Brasil. Forma Biológica: 1= Árvore, 2= Arbusto, 3= Palmeira. As fontes estão devidamente listadas na tabela 1. Referencias em Herbário: ESA= Herbário da Escola Superior de Agricultura Luiz de Queiroz-ESALQ. Piracicaba, SP.; CGMS= Herbário da Universidade Federal de Mato Grosso do Sul, Campo Grande, MS.

\begin{tabular}{|c|c|c|c|c|c|}
\hline Família & Espécie & Nome popular & $\begin{array}{c}\text { Forma } \\
\text { biológica }\end{array}$ & Fonte & $\begin{array}{l}\text { Referência em } \\
\text { Herbário }\end{array}$ \\
\hline \multirow[t]{2}{*}{ Acanthaceae } & Ruellia erythropus (Nees) Lindau & & 2 & 1 & 51621 - ESA \\
\hline & R. gemminiflora Kunth & & 2 & $5,7,9,10$ & \\
\hline \multirow[t]{11}{*}{ Anacardiaceae } & Anacardium occidentale L. & Caju & 1 & 7 & \\
\hline & A. humile A. A. St.Hil. & Caju-rasteiro & 2 & 7 & \\
\hline & Astronium fraxinifolium Schott ex Spreng. & Gonçalo & 1 & $\begin{array}{c}1,4,5,6,9,10,11,12,13 \\
14,15\end{array}$ & 51625 - ESA \\
\hline & Astronium graveolens Jacq. & Guarita & 1 & $\begin{array}{c}1,2,4,5,6,9,10,11,12 \\
13,14,15\end{array}$ & $\begin{array}{c}51626-\text { ESA } \\
12928-\text { CGMS }\end{array}$ \\
\hline & Lithraea molleoides (Vell.) Engl. & Aroeira-branca & 1 & $\begin{array}{c}1,4,5,6,8,9,10,11,12 \\
14,15\end{array}$ & 51627 - ESA \\
\hline & Myracrodruon urundeuva Allemão & Aroeira & 1 & $\begin{array}{c}1,3,4,5,6,7,8,910,11 \\
12,13,14,15\end{array}$ & 51628 - ESA \\
\hline & Schinopsis balansae Engl. & Quebracho & 1 & $6,7,10$ & \\
\hline & Schinus terebinthifolius Raddi & Aroeira-pimenteira & 1 & $\begin{array}{c}1,4,5,6,7,8,9,1011 \\
14,15\end{array}$ & 51629 - ESA \\
\hline & S. weinmaniifolius Engl. & Aroeirinha & 2 & $7,8,9$ & \\
\hline & Spondias mombin L. (lutea) & Cajá & 1 & $6,9,15$ & \\
\hline & Tapirira guianensis Aubl. & Peito-de-pomba & 1 & $\begin{array}{c}1,2,4,5,6,7,8,9,10,11 \\
12,13,14,15\end{array}$ & $\begin{array}{c}51630 \text { - ESA; } \\
\text { 17299, } 12929 \text { - } \\
\text { CGMS }\end{array}$ \\
\hline \multirow[t]{9}{*}{ Annonaceae } & Annona cacans Warm. & Cortição & 1 & 14,15 & \\
\hline & A. coriacea Mart. & Marolo & 1 & $1,4,5,6,7,8,9,10,13$ & 51631 - ESA \\
\hline & A. cornifolia A. St. Hill. & Ariticum-de-cobra & 2 & $5,6,7,8,9,10,13,15$ & \\
\hline & A. crassiflora Mart. & Araticum-do-cerrado & 1 & $4,5,7,8,9,10,13$ & \\
\hline & A. dioica A. A. St.Hil. & Araticum & 1 & $4,8,9$ & \\
\hline & A. emarginata (Schltdl.) H.Rainer & Araticum-do-mato & 1 & $2,5,6,7,10,13,14,15$ & 12930 - CGMS \\
\hline & A. phaeoclados Mart. & Araticum & 1 & 7 & \\
\hline & A. sylvatica A.St.-Hil. & Araticum-do-mato & 1 & $4,7,13,14$ & \\
\hline & A. warmingiana Mello-Silva \& Pirani & Araticum-rasteiro & 2 & 7 & \\
\hline \multirow[t]{6}{*}{ Annonaceae } & Duguetia furfuracea (A. A. St.Hil.) Saff. & Ata brava & 2 & $4,5,6,7,8,9,10,13$ & \\
\hline & D. lanceolata A. St.Hil. & Pindaíva & 1 & 13,14 & \\
\hline & Unonopsis guatterioides (A.DC.) R.E.Fr. & $\begin{array}{l}\text { Pindaíva, pindaíba- } \\
\text { do-brejo }\end{array}$ & 1 & $\begin{array}{c}1,2,3,5,6,8,9,10,11 \\
12,14,15\end{array}$ & $\begin{array}{l}51632 \text { - ESA; } \\
12931 \text { - CGMS }\end{array}$ \\
\hline & Xylopia aromatica (Lam.) Mart. & Pimenta-de-macaco & 1 & $1,4,5,6,7,8,10,11,13$ & $\begin{array}{l}51633 \text { - ESA; } \\
17423 \text { - CGMS }\end{array}$ \\
\hline & X. emarginata Mart. & Pindaíba-d'-água & 1 & $4,5,10,13$ & \\
\hline & X. brasiliensis Spreng. & Pindaíba & 1 & 14 & \\
\hline \multirow[t]{8}{*}{ Apocynaceae } & Aspidosperma australe Müll.Arg. & Guatambu & 1 & 4 & \\
\hline & A. cuspa (Kunth.) S.F. Blake ex Pittier & Guatambu branco & 1 & $\begin{array}{c}1,4,5,6,8,9,10,11,12 \\
13,14,15\end{array}$ & 51634 - ESA \\
\hline & A. cylindrocarpon Müll.Arg. & Peroba - poca & 1 & $\underset{15}{1,5,6,9,10,11,12,14}$ & $\begin{array}{l}51635 \text { - ESA; } \\
17301 \text { - CGMS }\end{array}$ \\
\hline & A. macrocarpon Mart. & Guatambu-do-cerrado & 1 & $4,5,6,7,10,13,15$ & \\
\hline & A. parvifolium A.DC. & $\begin{array}{l}\text { Guatambu-oliva, } \\
\text { guatambu-amarelo }\end{array}$ & 1 & $\begin{array}{c}1,2,5,6,10,11,12,14 \\
15\end{array}$ & $\begin{array}{l}51636 \text { - ESA; } \\
12958 \text { - CGMS }\end{array}$ \\
\hline & A. polyneuron Müll.Arg. & Peroba - rosa & 1 & $\begin{array}{c}1,5,6,9,10,11,12,14 \\
15\end{array}$ & 51637 - ESA \\
\hline & A. pyrifolium Mart. & $\begin{array}{l}\text { Pau-pereiro, peroba- } \\
\text { branca }\end{array}$ & 1 & $2,4,5,6,10,11,12,14$ & 12932 - CGMS \\
\hline & A. quebracho-blanco Schltdl. & Quebracho-branco & 1 & 6,11 & \\
\hline
\end{tabular}


Quadro 2. Cont.

\begin{tabular}{|c|c|c|c|c|c|}
\hline Família & Espécie & Nome popular & $\begin{array}{l}\text { Forma } \\
\text { biológica }\end{array}$ & Fonte & $\begin{array}{l}\text { Referência em } \\
\text { Herbário }\end{array}$ \\
\hline \multirow{8}{*}{ Araliaceae } & A. subincanum Mart. & Guatambu-vermelho & 1 & $\begin{array}{c}1,4,5,6,9,10,11,12,13 \\
14,15\end{array}$ & 51638 - ESA \\
\hline & A. tomentosum Mart. & Peroba-do-campo & 1 & $4,5,6,7,8,10,11,13$ & \\
\hline & Hancornia speciosa Gomes & Mangaba & 1 & $5,7,10,13$ & \\
\hline & Himatanthus obovatus (Müll.Arg.) Woodson & Pau-de-leite & 1 & 4,13 & \\
\hline & Thevetia bicornuta Müll.Arg. & Leiteirinho & 2 & 6,11 & \\
\hline & $\begin{array}{l}\text { Dendropanax cuneatus (DC.) Decne. \& } \\
\text { Planch. }\end{array}$ & & 1 & $\begin{array}{c}1,4,5,6,8,9,10,11,12 \\
13,14\end{array}$ & $\begin{array}{l}51643,51642- \\
\text { ESA }\end{array}$ \\
\hline & $\begin{array}{l}\text { Schefflera macrocarpa (Cham. \& Schltdl.) } \\
\text { Frodin. }\end{array}$ & $\begin{array}{l}\text { Mandiocão-do- } \\
\text { cerrado }\end{array}$ & 1 & 4,7 & \\
\hline & $\begin{array}{l}\text { S. morototoni (Aubl.) Maguire, Steyerm. } \\
\text { \& Frodin. }\end{array}$ & Mandiocão & 1 & $\begin{array}{c}1,4,5,6,7,8,10,11 \\
13,14\end{array}$ & 51644 - ESA \\
\hline \multirow[t]{13}{*}{ Arecaceae } & Acrocomia aculeata (Jacq.) Lodd. ex Mart. & Bocaiúva & 3 & $\begin{array}{c}1,4,5,6,7,8,9,10,11 \\
12,13,14,15\end{array}$ & 51645 - ESA \\
\hline & Allagoptera leucocalyx (Drude.) Kuntze & Iriri & 3 & $5,6,7,10,13$ & \\
\hline & Astrocaryum vulgare Mart. & Tucum & 3 & 10 & \\
\hline & Attalea oleifera Barb. Rodr. & Babaçu & 3 & 10,13 & \\
\hline & A. phalerata Mart. ex Spreng. & Bacuri, acuri & 3 & $\begin{array}{c}1,3,4,5,6,9,10,11,12 \\
14,15\end{array}$ & 51646 - ESA \\
\hline & Bactris glaucescens Drude & Tucum & 3 & 6,10 & \\
\hline & Butia paraguayensis (Barb. Rodr.) Bailey & Cabeçudinha & 3 & $4,5,6,7,8,10,13$ & \\
\hline & $\begin{array}{l}\text { Copernicia alba Morong ex Morong \& } \\
\text { Britton }\end{array}$ & Carandá & 3 & 6,11 & \\
\hline & Mauritia flexuosa L.f. & Buriti & 3 & 4,10 & \\
\hline & Syagrus flexuosa (Mart.) Becc. & Acumã & 3 & 10,13 & \\
\hline & S. petraea Becc. & Iriri & 3 & 7 & \\
\hline & S. romanzoffiana (Cham.) Glassman & Pindó, jerivá & 3 & $\begin{array}{c}1,5,6,7,8,10,11,12,13 \\
14,15\end{array}$ & 51647 - ESA \\
\hline & S. oleracea (Mart.) Becc. & Gariroba & 3 & $5,13,15$ & \\
\hline \multirow[t]{9}{*}{ Asteraceae } & Baccharis dracunculifolia DC. & Vassoura & 2 & $4,5,7,8,9,10,13,15$ & \\
\hline & Dasyphyllum brasiliense (Spreng.) Cabrera & Espinho-Agulha & 2 & $4,5,8,9,10,12,13,14$ & \\
\hline & $\begin{array}{l}\text { Chromolaena maximilianii (Schrad. ex DC.) } \\
\text { R.M.King \& H.Rob. }\end{array}$ & & 2 & 9 & \\
\hline & C. orbignyana (Klatt) R.M.King \& H.Rob. & & 2 & 9 & \\
\hline & Eremanthus glomerulatus Less. & Coração-de-negro & 2 & $5,7,8,10,13,15$ & \\
\hline & Lessingianthus glaziovianus (Baker) H.Rob. & Assa-peixe & 2 & $5,10,12,13,14,15$ & \\
\hline & Vernonanthura ferruginea (Less.) H.Rob. & Calção-de-velho & 2 & $\begin{array}{c}4,5,6,7,8,9,10,11 \\
13,15\end{array}$ & \\
\hline & V. phosphorica (Vell.) H.Rob. & Assa-peixe & 2 & $5,10,13,15$ & \\
\hline & Vernonia scabra Pers. & Assa-peixe & 2 & $\begin{array}{c}1,4,5,6,7,8,9,10,11 \\
12,13,14,15\end{array}$ & $\begin{array}{l}51655 \text { - ESA; } \\
17309-\text { CGMS }\end{array}$ \\
\hline \multirow[t]{7}{*}{ Bignoniaceae } & Cybistax antisyphilitica (Mart.) Mart. & Ipê-Verde & 1 & 4 & \\
\hline & Handroanthus albus (Cham.) Mattos & Ipê-amarelo & 1 & $12,14,15$ & \\
\hline & H. heptaphyllus (Vell.) Mattos & Piúva & 1 & $\begin{array}{c}1,5,6,8,9,10,11,12 \\
14,15\end{array}$ & 51662 - ESA \\
\hline & H. impetiginosus (Mart. ex DC.) Mattos & $\begin{array}{l}\text { Ipê roxo, piúva-da- } \\
\text { mata }\end{array}$ & 1 & $\begin{array}{l}1,2,5,6,7,8,9,10,11 \\
12,13,14,15\end{array}$ & $\begin{array}{c}51663 \text { - ESA; } \\
\text { 17427, } 12933- \\
\text { CGMS }\end{array}$ \\
\hline & H. ochraceus (Cham.) Mattos & Ipê-amarelo & 1 & $\begin{array}{c}1,4,5,6,7,8,10,11,12 \\
13,14,15\end{array}$ & $\begin{array}{l}51664 \text { - ESA; } \\
17311 \text { - CGMS }\end{array}$ \\
\hline & H. serratifolius (A.H.Gentry) S.Grose & Pau-d'arco & 1 & $4,5,8,10,13,15$ & \\
\hline & H. umbellatus (Sond.) Mattos & Ipê-amarelo & 1 & 3 & \\
\hline
\end{tabular}


Quadro 2. Cont.

\begin{tabular}{|c|c|c|c|c|c|}
\hline Família & Espécie & Nome popular & $\begin{array}{l}\text { Forma } \\
\text { biológica }\end{array}$ & Fonte & $\begin{array}{l}\text { Referência em } \\
\text { Herbário }\end{array}$ \\
\hline & Jacaranda brasiliana (Lam.) Pers. & Boca-de-sapo & 1 & $5,6,10,11,13$ & \\
\hline & J. cuspidifolia Mart. & Caroba & 1 & $\begin{array}{c}1,4,5,6,7,8,9,10,11 \\
12,13,14,15\end{array}$ & 51660 - ESA \\
\hline & J. decurrens $\mathrm{Mez}$ & Carobinha & 2 & 7 & \\
\hline & J. micrantha Cham. & Caroba & 1 & $7,12,14,15$ & \\
\hline & $\begin{array}{l}\text { Tabebuia aurea (Silva Manso) Benth. \& } \\
\text { Hook.f. ex S.Moore }\end{array}$ & Ipê-amarelo & 1 & $3,4,5,6,7,8,10,11,13$ & \\
\hline & T. insignis (Miq.) Sandwith & Ipê-amarelo & 1 & $4,10,15$ & 17428 - CGMS \\
\hline & T. nodosa (Griseb.) Griseb. & Labão & 1 & 6,11 & \\
\hline & T. roseoalba (Ridl.) Sandwith & Ipê-branco & 1 & $\begin{array}{c}1,2,4,5,8,9,10,11,12 \\
13,15\end{array}$ & $\begin{array}{l}51665 \text { - ESA; } \\
12934 \text { - CGMS }\end{array}$ \\
\hline & Zeyheria montana Mart. & Bolsinha-de-pastor & 1 & 4,7 & \\
\hline \multirow[t]{2}{*}{ Bixaceae } & Bixa orellana $\mathrm{L}$. & Urucum, colorau & 1 & $1,4,5,6,7,10,11,13$ & 51666 - ESA \\
\hline & $\begin{array}{l}\text { Cochlospermum regium } \\
\text { (Mart. ex Schrank) Pilg. }\end{array}$ & $\begin{array}{l}\text { Algodãozinho-do- } \\
\text { cerrado }\end{array}$ & 2 & 7,10 & \\
\hline \multirow[t]{6}{*}{ Boraginaceae } & Cordia alliodora (Ruiz \& Pav.) Cham. & Falso-louro & 1 & 4 & \\
\hline & C. americana (L.) Gottschling \& J.S.Mill. & Guajuvira & 1 & $\begin{array}{c}1,2,4,5,6,8,9,10,11 \\
12,14,15\end{array}$ & $\begin{array}{l}51671 \text { - ESA; } \\
12935,17312- \\
\text { CGMS }\end{array}$ \\
\hline & $\begin{array}{l}\text { C. brasiliensis (I.M.Johnst.) Gottschling } \\
\text { \& J.S.Mill. }\end{array}$ & Louro-de-capote & 1 & 6,11 & \\
\hline & C. glabrata (Mart.) A. DC. & Louro-preto & 1 & $\begin{array}{c}1,4,5,6,7,8,9,10,12 \\
13,14,15\end{array}$ & $\begin{array}{l}51667 \text { - ESA; } \\
17315 \text { - CGMS }\end{array}$ \\
\hline & C. sellowiana Cham. & $\begin{array}{l}\text { Chá-de-bugre, louro- } \\
\text { preto }\end{array}$ & 1 & $\begin{array}{c}1,2,4,5,6,9,10,11,12 \\
13,14\end{array}$ & $\begin{array}{l}51668 \text { - ESA; } \\
12935,17314- \\
\text { CGMS }\end{array}$ \\
\hline & C. trichotoma (Vell.) Arráb. ex Steud. & Louro-pardo & 1 & $\begin{array}{c}1,4,5,6,10,11,12,13 \\
14,15\end{array}$ & $\begin{array}{l}51670 \text { - ESA; } \\
17313,17429- \\
\text { CGMS }\end{array}$ \\
\hline \multirow[t]{2}{*}{ Burseraceae } & Protium heptaphyllum (Aubl.) Marchand & Almecega, breu & 1 & $\begin{array}{c}1,4,5,6,7,8,9,10,11 \\
12,13,14,15\end{array}$ & $51673-\mathrm{ESA}$ \\
\hline & P. spruceanum (Benth.) Engl. & Breu & 1 & 4 & \\
\hline \multirow[t]{2}{*}{ Cactaceae } & Cereus bicolor Rizzini \& A.Mattos & Cacto & 1 & 6 & \\
\hline & C. hildmannianus K. Schum. & Mandacaru & 1 & $4,5,6,7,8,10,11,13,15$ & \\
\hline Calophyllaceae & Calophyllum brasiliense Cambess. & Guanandi & 1 & $2,4,5,6,10,12,14$ & \\
\hline \multirow[t]{5}{*}{ Cannabaceae } & Celtis fluminensis Carauta & Esporão-de-galo & 1 & 14 & \\
\hline & C. iguanaea (Jacq.) Sarg. & Grão-de-galo & 2 & $\begin{array}{c}1,5,6,7,8,9,10,11,12 \\
13,14,15\end{array}$ & 51674 - ESA \\
\hline & C. pubescens (Kunt) Spreng. & Taleira & 2 & $\begin{array}{c}1,2,4,5,6,7,8,9,10,11 \\
14,15\end{array}$ & $\begin{array}{l}51675-\text { ESA; } \\
17430,12961- \\
\text { CGMS }\end{array}$ \\
\hline & Celtis spinosa Spreng. & Gurupiá & 1 & $\begin{array}{c}1,4,5,6,9,10,11,12 \\
14,15\end{array}$ & 51677 - ESA \\
\hline & Trema micrantha (L.) Blume & Grandiúva & 1 & $\begin{array}{c}1,2,4,5,6,8,9,10,11 \\
12,13,14,15\end{array}$ & $\begin{array}{l}51678 \text { - ESA; } \\
\text { 12960, } 17317- \\
\text { CGMS }\end{array}$ \\
\hline \multirow[t]{2}{*}{ Capparaceae } & $\begin{array}{l}\text { Anisocapparis speciosa (Griseb.) } \\
\text { Cornejo \& Iltis }\end{array}$ & Mangava-brava & 1 & 6,11 & \\
\hline & $\begin{array}{l}\text { Capparicordis tweediana (Eich.) } \\
\text { Iltis \& Cornejo }\end{array}$ & & 2 & 11 & \\
\hline
\end{tabular}


Quadro 2. Cont.

\begin{tabular}{|c|c|c|c|c|c|}
\hline Família & Espécie & Nome popular & $\begin{array}{l}\text { Forma } \\
\text { biológica }\end{array}$ & Fonte & $\begin{array}{l}\text { Referência em } \\
\text { Herbário }\end{array}$ \\
\hline & Capparidastrum petiolare (Kunth) Hutch. & & & $1,4,5,10$ & 51672 - ESA \\
\hline & Crataeva tapia $\mathrm{L}$. & Cabaceira & 1 & 6,11 & \\
\hline & Cynophalla retusa (Griseb.) Cornejo \& Iltis & & 1 & $2,6,11$ & \\
\hline \multirow[t]{2}{*}{ Caricaceae } & Jacaratia spinosa (Aubl.) A. DC. & $\begin{array}{l}\text { Jaracatia, mamão-do- } \\
\text { mato }\end{array}$ & 1 & $\begin{array}{c}1,2,5,6,8,10,11,12 \\
14,15\end{array}$ & $\begin{array}{l}51679 \text { - ESA; } \\
12951 \text { - CGMS }\end{array}$ \\
\hline & J. corumbensis Kuntze & Mamãozinho & 1 & 11 & \\
\hline Caryocaraceae & Caryocar brasiliense Cambess. & Pequi & 1 & $4,5,6,7,10,13$ & \\
\hline \multirow[t]{5}{*}{ Celastraceae } & Maytenus ilicifolia Mart. ex Reissek & Espinheira-santa & 2 & $1,5,6,10,11$ & 51681 - ESA \\
\hline & M. macrodonta Reissek & & 2 & 1 & 51682 - ESA \\
\hline & Plenckia populnea Reissek & Marmelo-do-cerrado & 1 & 13 & \\
\hline & Salacia elliptica (Mart. ex Schult.) G. Don & Saputá & 1 & $\begin{array}{c}1,2,4,5,6,8,9,10,11 \\
15\end{array}$ & $\begin{array}{l}51683 \text { - ESA; } \\
12944 \text { - CGMS }\end{array}$ \\
\hline & S. crassifolia (Mart. ex Schult.) G.Don & Bacupari-do-cerrado & 1 & 4,13 & \\
\hline \multirow[t]{4}{*}{ Chrysobalanacea } & e Couepia grandiflora (Mart. et Zucc.) Benth. & $\begin{array}{l}\text { Genciana, oiti-do- } \\
\text { cerrado }\end{array}$ & 1 & $4,5,7,10,13,15$ & \\
\hline & Hirtella gracilipes (Hook.f.) Prance & Bosta-de-cabra & 1 & 13 & \\
\hline & Licania minutiflora (Sagot) Fritsch & Cedro-d'água & 1 & $4,5,10$ & \\
\hline & L. tomentosa (Benth.) Fritsch & Oiti & 1 & 13 & \\
\hline \multirow[t]{6}{*}{ Clusiaceae } & $\begin{array}{l}\text { Garcinia gardneriana (Planch. \& Triana) } \\
\text { Zappi }\end{array}$ & Bacupari & 1 & $1,6,11$ & 51684 - ESA \\
\hline & $\begin{array}{l}\text { Rheedia brasiliensis (Mart.) Planch. \& } \\
\text { Triana }\end{array}$ & Bacupari & 1 & $5,10,15$ & \\
\hline & Kielmeyera coriacea Mart. \& Zucc. & Gordiana, pau-santo & 1 & $4,5,6,7,10,13$ & \\
\hline & K. rubriflora Cambess. & Rosa-do-cerrado & 1 & $5,10,13$ & \\
\hline & K. speciosa A.-St.Hil. & Pau-santo & 1 & 13 & \\
\hline & K. variabilis Mart. \& Zucc. & Pau-santo & 1 & 7 & \\
\hline \multirow[t]{2}{*}{ Combretaceae } & Buchenavia tomentosa Eichler & Tarumarana & 1 & $4,5,6,8,10,13$ & \\
\hline & Combretum duarteanum Cambess. & Pombeiro & 1 & $\begin{array}{c}4,5,6,10,11,12,13 \\
14,15\end{array}$ & \\
\hline \multirow[t]{6}{*}{ Combretaceae } & C. leprosum Mart. & Carne-de-vaca & 1 & $\begin{array}{c}1,2,4,5,6,9,10,11,12 \\
13,14,15\end{array}$ & $\begin{array}{c}51686 \text { - ESA; } \\
\text { 12949, } 17319 \text { - } \\
\text { CGMS }\end{array}$ \\
\hline & C. mellifluum Eichler & Pombeiro-do-cerrado & 1 & 1,4 & $\begin{array}{l}51685 \text { - ESA; } \\
17320 \text { - CGMS }\end{array}$ \\
\hline & Terminalia argentea Mart. & Capitão & 1 & $\begin{array}{c}1,4,5,6,7,9,10,11 \\
13,15\end{array}$ & 51687 - ESA \\
\hline & T. fagifolia Mart. \& Zucc. & Orelha-de-cachorro & 1 & 3,13 & \\
\hline & T. glabrescens Mart. & Capitão-do-campo & 1 & $10,12,14$ & \\
\hline & T. triflora (Griseb.) Lillo & Alazão & 1 & $\begin{array}{c}1,5,6,9,10,12,13,14 \\
15\end{array}$ & $\begin{array}{c}51689- \\
\text { ESA;17318 - } \\
\text { CGMS }\end{array}$ \\
\hline \multirow[t]{2}{*}{ Connaraceae } & Connarus suberosus Planch. & Araruta-do-campo & 1 & $4,5,7,10,13$ & \\
\hline & Rourea induta Planch. & Botica & 1 & 13 & \\
\hline \multirow[t]{2}{*}{ Dilleniaceae } & Curatella americana $L$. & Lixeira & 1 & $1,4,5,6,8,10,13$ & 51696 - ESA \\
\hline & Davilla elliptica A.St.-Hill. & Lixeirinha & 1 & $4,5,7,8,10,13,15$ & 17433 - CGMS \\
\hline Ebenaceae & Diospyros hispida A. DC. & Fruta-de-boi & 1 & 4 & \\
\hline Elaeocarpaceae & Sloanea hirsuta (Schott) Planch. ex Benth. & Ouriço & 1 & 13,14 & 17434 - CGMS \\
\hline \multirow[t]{2}{*}{ Erythroxylaceae } & Erythroxylum anguifugum Mart. & Pimenteirinha & 2 & 6,11 & \\
\hline & E. campestre A. St.Hil. & Cabelo-de-negro & 2 & 7 & \\
\hline
\end{tabular}


Quadro 2. Cont.

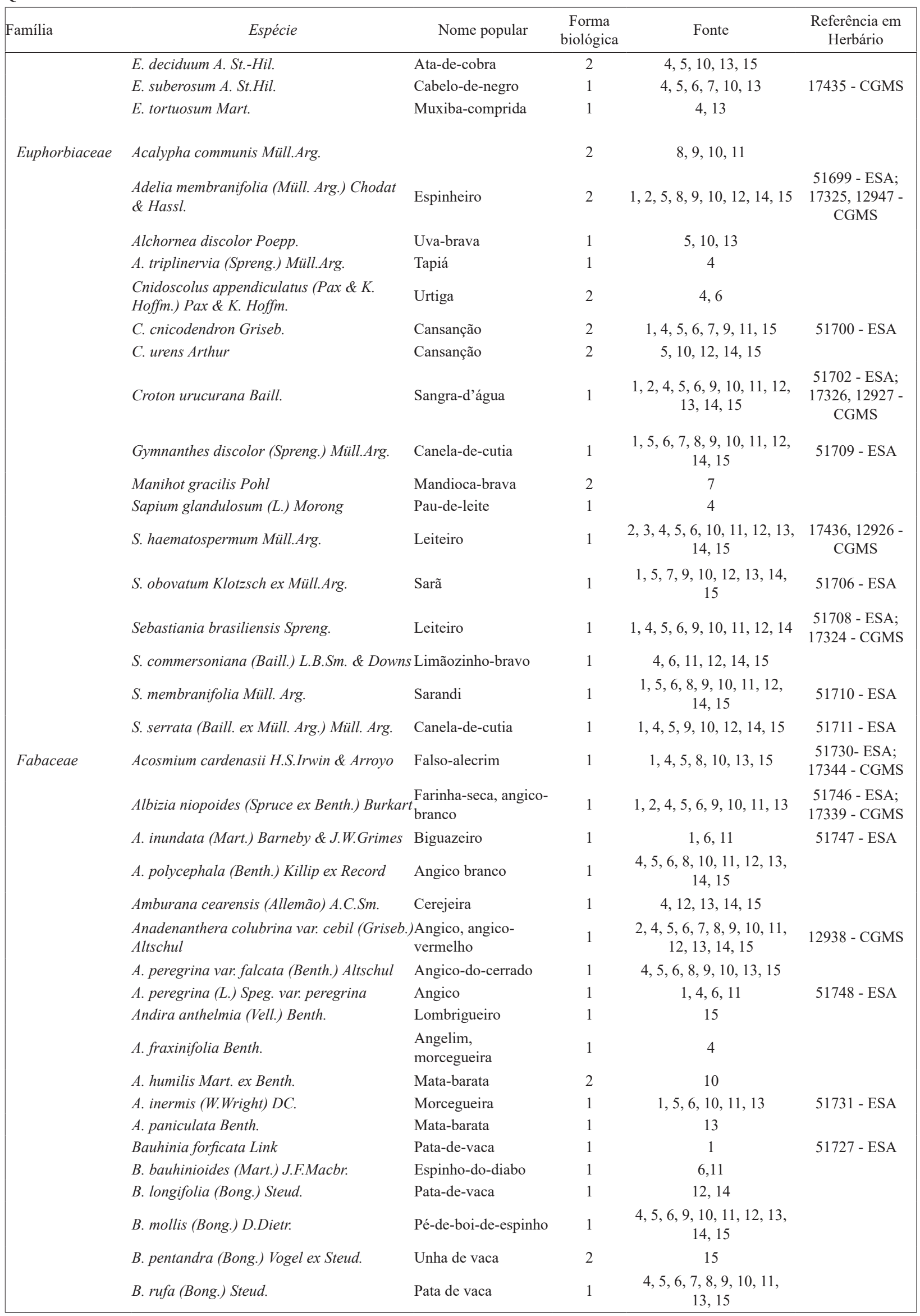


Quadro 2. Cont.

\begin{tabular}{|c|c|c|c|c|c|}
\hline Família & Espécie & Nome popular & $\begin{array}{l}\text { Forma } \\
\text { biológica }\end{array}$ & Fonte & $\begin{array}{l}\text { Referência em } \\
\text { Herbário }\end{array}$ \\
\hline & Bowdichia virgilioides Kunth. & Sucupira-preta & 1 & $4,5,6,7,10,13,15$ & \\
\hline & $\begin{array}{l}\text { Caesalpinia paraguariensis (Parodi) } \\
\text { Burkart }\end{array}$ & Pau-ferro & 1 & 6,11 & \\
\hline & Calliandra dysantha Benth. & Cigana & 2 & 7 & \\
\hline & C. parviflora (Hook. \& Arn.) Speg. & Angiquinho & 2 & $1,4,5,6,9,10,13$ & $\begin{array}{l}51750-\text { ESA; } \\
17438-\text { CGMS }\end{array}$ \\
\hline & Cassia ferruginea (Schrad.) Schrad. ex DC. & Chuva-de-ouro & 1 & 6 & \\
\hline & C. grandis L.f. & Cassia-grande & 1 & 6,15 & \\
\hline & $\begin{array}{l}\text { Chamaecrista orbiculata (Benth.) H.S.Irwin } \\
\text { \& Barneby }\end{array}$ & Planta-moeda & 2 & 7 & \\
\hline & Chloroleucon tortum (Mart.) Pittier & Barreiro & 1 & 6,11 & \\
\hline & Copaifera langsdorffii Desf. & Copaíba, pau-óleo & 1 & $4,5,6,7,8,10,13$ & \\
\hline & Dalbergia nigra (Vell.) Allemão ex Benth. & Jacarandá & 1 & 4 & \\
\hline & D. miscolobium Benth. & Jacarandá-miúdo & 1 & $4,7,13$ & \\
\hline & Dimorphandra mollis Benth. & Faveira-do-campo & 1 & $4,5,6,7,8,10,13$ & \\
\hline & Diptychandra aurantiaca Tul. & Carvão-vermelho & 1 & $1,4,5,6,7,8,10,13,15$ & $\begin{array}{c}51713 \text { - ESA; } \\
17335,17439 \\
17445 \text { - CGMS }\end{array}$ \\
\hline & Dipteryx alata Vogel & Cumaru, baru & 1 & $\begin{array}{c}1,3,4,5,6,7,8,10,13 \\
14,15\end{array}$ & 51734 - ESA \\
\hline & $\begin{array}{l}\text { Enterolobium contortisiliquum (Vell.) } \\
\text { Morong }\end{array}$ & $\begin{array}{l}\text { Ximbuva, orelha-de- } \\
\text { negro }\end{array}$ & 1 & $\begin{array}{c}1,4,5,6,8,9,10,11,12 \\
13,14,15\end{array}$ & 51752 - ESA \\
\hline & Erythrina verna Vell. & Mulungu-coral & 1 & $4,5,6,10,13$ & \\
\hline & $\begin{array}{l}\text { Guibourtia hymenaeifolia (Moric.) } \\
\text { J.Léonard }\end{array}$ & Jatobá-mirim & 1 & $\begin{array}{c}1,2,5,6,8,9,10,11,12 \\
13,14,15\end{array}$ & $\begin{array}{l}51714 \text { - ESA; } \\
12941 \text { - CGMS }\end{array}$ \\
\hline & Holocalyx balansae Micheli & Alecrim & 1 & $\begin{array}{c}1,2,4,5,6,8,9,10,11 \\
12,14,15\end{array}$ & $\begin{array}{l}51715 \text { - ESA; } \\
12936 \text { - CGMS }\end{array}$ \\
\hline & Hymenaea courbaril L. & Jatobá & 1 & $\begin{array}{c}1,4,5,6,8,9,10,11,12 \\
13,14,15\end{array}$ & 51717 - ESA \\
\hline & H. stigonocarpa Mart. ex Hayne & Jatobá-do-cerrado & 1 & $1,4,5,6,7,8,10,13$ & $\begin{array}{l}51716 \text { - ESA; } \\
17330 \text { - CGMS }\end{array}$ \\
\hline & Inga edulis Mart. & Ingá & 1 & 1,4 & $\begin{array}{l}51755 \text { - ESA; } \\
17441 \text { - CGMS }\end{array}$ \\
\hline & I. heterophylla Willd. & Ingá & 1 & 5,10 & \\
\hline & I. laurina (Sw.) Willd. & Ingá-branco & 1 & $1,5,6,9,10,12,14,15$ & 51754 - ESA \\
\hline & I. marginata Willd. & Ingá-do-brejo & 1 & $\begin{array}{c}1,2,4,5,6,9,10,11,12 \\
13,14,15\end{array}$ & $\begin{array}{l}51753 \text { - ESA; } \\
12937 \text { - CGMS }\end{array}$ \\
\hline & I. umbellata (Vahl) Willd. & Ingá & 1 & 3 & \\
\hline & I. vera subsp. affinis (DC.) T.D.Penn. & Ingá-do-brejo & 1 & $\begin{array}{c}1,3,4,5,6,7,9,10,12 \\
13,14,15\end{array}$ & $\begin{array}{l}51757 \text { - ESA; } \\
17337 \text { - CGMS }\end{array}$ \\
\hline & Leptolobium dasycarpum Vogel & & 1 & $4,5,7,10,13,14,15$ & \\
\hline & L. elegans Vogel & Quina-genciana & 1 & $4,5,6,7,10,13,14,15$ & \\
\hline & $\begin{array}{l}\text { Lonchocarpus cultratus (Vell.) } \\
\text { A.M.G.Azevedo \& H.C.Lima }\end{array}$ & Rabo-de-bugio & 1 & 1 & 51735 - ESA \\
\hline & L. muehlbergianus Hassl. & Timbó & 1 & $5,10,13,15$ & 17442 - CGMS \\
\hline & L. sericeus (Poir.) Kunth ex DC. & Falso-ingá & 1 & $1,4,6,11$ & 51736 - ESA \\
\hline & Machaerium aculeatum Raddi & Barreiro & 1 & $\begin{array}{c}5,6,8,9,10,11,12,13 \\
14,15\end{array}$ & \\
\hline & M. acutifolium Vogel & Jacarandá & 1 & $\begin{array}{c}1,4,5,6,7,9,10,11,12 \\
13,14,15\end{array}$ & 51737 - ESA \\
\hline & M. eriocarpum Benth. & Jacarandá & 1 & 1 & 51738 - ESA \\
\hline & M. hirtum (Vell.) Stellfeld & Jacarandá & 1 & $1,6,9,11$ & $\begin{array}{l}\text { 51739- ESA; } \\
17334 \text {-CGMS }\end{array}$ \\
\hline & M. isadelphum (E. Mey.) Amshoff & Jacarandá & 1 & 1 & 51740 - ESA \\
\hline & M. opacum Vog. & Jacarandá-do-cerrado & 1 & 4,7 & \\
\hline & M. scleroxylon Tul. & Caviúma & 1 & 14,15 & \\
\hline & M. stipitatum Vogel & Sapuvinha & 1 & 14,15 & \\
\hline
\end{tabular}


Quadro 2. Cont.

\begin{tabular}{|c|c|c|c|c|c|}
\hline Família & Espécie & Nome popular & $\begin{array}{c}\text { Forma } \\
\text { biológica }\end{array}$ & Fonte & $\begin{array}{l}\text { Referência em } \\
\text { Herbário }\end{array}$ \\
\hline & M. villosum Vogel & Jacarandá & 1 & $1,12,14,15$ & 51741 - ESA \\
\hline & $\begin{array}{l}\text { Microlobius foetidus var. paraguensis } \\
\text { (Benth.) M.Sousa \& G.Andrade }\end{array}$ & Pau-alho & 1 & 6,11 & \\
\hline & Mimosa adenocarpa Benth. & Arranha-gato & 2 & 6,11 & \\
\hline & M. chaetosphaera Barn. & Espinheiro-preto & 2 & $6,11,13$ & \\
\hline & M. claussenii Benth. & Mimosa & 2 & $5,7,9,10,12,13,14,15$ & \\
\hline & M. glutinosa Malme & Espinilho & 1 & $1,6,8,11$ & 51758 - ESA \\
\hline & M. hexandra Micheli & Espinheiro & 2 & 11 & \\
\hline & M. setosa Benth. & Espinheiro & 2 & 4 & \\
\hline & $\begin{array}{l}\text { Muellera sericea (Micheli) M.J. Silva } \\
\& \text { A.M.G. Azevedo }\end{array}$ & Biuera & 1 & 11 & \\
\hline & Myroxylon peruiferum L.f. & Bálsamo & 1 & $1,2,5,8,10,14,15$ & $\begin{array}{c}51742 \text { - ESA; } \\
\text { 17342, } 12959- \\
\text { CGMS }\end{array}$ \\
\hline & Parapiptadenia rigida (Benth.) Brenan & Angico-da-mata & 1 & $\begin{array}{c}1,2,4,5,6,8,9,10,11 \\
12,14,15\end{array}$ & $\begin{array}{c}51761 \text { - ESA; } \\
\text { 17341, } 12939- \\
\text { CGMS }\end{array}$ \\
\hline & Peltogyne angustiflora Ducke & Roxinho & 1 & $\begin{array}{c}4,5,6,7,8,10,11,13 \\
14,15\end{array}$ & \\
\hline & Peltophorum dubium (Spreng.) Taub. & Canafístula & 1 & $\begin{array}{c}1,4,5,6,8,9,10,12 \\
14,15\end{array}$ & $\begin{array}{l}\text { 51718- ESA; } \\
17333 \text { - CGMS }\end{array}$ \\
\hline & Piptadenia gonoacantha (Mart.) J.F.Macbr. & Pau-jacaré & 1 & $5,6,8,9, \underset{15}{10}, 11,12,14$ & \\
\hline & P. viridiflora (Kunth) Benth. & Espinheiro & 1 & $12,14,15$ & \\
\hline & Plathymenia reticulata Bth. & Vinhatico & 1 & $4,5,6,7,8,10,13$ & \\
\hline & Platypodium elegans Vogel & Amendoim-do-campo & 1 & $1,5,6,7,9,10,11,14,15$ & 51743 - ESA \\
\hline & $\begin{array}{l}\text { Poincianella pluviosa var. peltophoroides } \\
\text { (Benth.) L.P.Queiroz }\end{array}$ & $\begin{array}{l}\text { Coração-de-negro, } \\
\text { sibipiruna }\end{array}$ & 1 & $4,5,6,9,10,11,12,13$ & $\begin{array}{l}51712 \text { - ESA; } \\
17336 \text { - CGMS }\end{array}$ \\
\hline & Prosopis rubriflora Hassl. & Espinheiro & 1 & 6,11 & \\
\hline & P. ruscifolia Gris & Algarobo & 1 & 6,11 & \\
\hline & Pterodon emarginatus Vog. & Faveiro & 1 & $4,5,6,10$ & \\
\hline & P. pubescens (Benth.) Benth. & Sucupira & 1 & 4 & \\
\hline & Pterogyne nitens Tul. & $\begin{array}{l}\text { Amendoim-do- } \\
\text { cerrado }\end{array}$ & 1 & $\begin{array}{c}1,2,3,4,5,6,7,8,9,10 \\
11,12,13,14,15\end{array}$ & $\begin{array}{l}51719-\text { ESA; } \\
17332-\text { CGMS }\end{array}$ \\
\hline & Riedeliella graciliflora Harms & Falsa ciganinha & 2 & 10,11 & \\
\hline & Samanea saman (Jacq.) Merr. & Sete-casca & 1 & $\begin{array}{c}5,6,9,10,11,12,13 \\
14,15\end{array}$ & \\
\hline & S. tubulosa (Benth.) Barneby \& J.W. Grimes & Alfarobo & 1 & $1,4,6,11,12,15$ & $\begin{array}{l}51762 \text { - ESA; } \\
17331 \text { - CGMS }\end{array}$ \\
\hline & Senegalia tenuifolia (L.) Britton \& Rose & Espinheiro & 1 & $4,6,11$ & \\
\hline & S. lowei (L.Rico) Seigler \& Ebinger & Arranha-gato & 2 & 9 & \\
\hline & S. polyphylla (DC.) Britton \& Rose & Monjoleiro & 1 & $\begin{array}{c}1,4,5,6,9,10,11,12 \\
14,15\end{array}$ & 51744 - ESA \\
\hline & $\begin{array}{l}\text { Senna aculeata (Pohl ex Benth.) H.S.Irwin } \\
\& \text { Barneby }\end{array}$ & Guelra-de-dourado & 2 & $5,6,7,9,10,11,13$ & \\
\hline & S. alata (L.) Roxb. & Mata-pasto & 2 & $1,4,5,6,9,10,11,15$ & 51720 - ESA \\
\hline & S. occidentalis (L.) Link & Fedegoso & 2 & $7,9,10,11,15$ & \\
\hline & $\begin{array}{l}\text { S. pendula (Humb.\& Bonpl.ex Willd.) } \\
\text { H.S.Irwin \& Barneby }\end{array}$ & & 1 & $1,7,10$ & 51721 - ESA \\
\hline & S. rugosa (G.Don) H.S.Irwin \& Barneby & Amarelinho & 2 & 7 & \\
\hline & $\begin{array}{l}\text { S. silvestris subsp. bifaria H.S.Irwin \& } \\
\text { Barneby }\end{array}$ & Fedegoso & 2 & 7 & \\
\hline & S. splendida (Vogel) H.S.Irwin \& Barneby & Fedegoso & 2 & 7,10 & \\
\hline & Sesbania sesban (L.) Merr. & & 1 & 1 & 51723 - ESA \\
\hline & \multicolumn{2}{|c|}{ Stryphnodendron adstringens (Mart.) CovilleBarbatimão } & 1 & $4,5,6,7,10,13$ & \\
\hline & Sweetia fruticosa Spreng. & Guaiçara & 1 & $5,10,14,15$ & \\
\hline
\end{tabular}


Quadro 2. Cont.

\begin{tabular}{|c|c|c|c|c|c|}
\hline Família & Espécie & Nome popular & $\begin{array}{l}\text { Forma } \\
\text { biológica }\end{array}$ & Fonte & $\begin{array}{l}\text { Referência em } \\
\text { Herbário }\end{array}$ \\
\hline & Tachigali aurea Tul. & Carvoeiro & 1 & $5,6,7,8,10,13$ & \\
\hline & T. vulgaris L.G.Silva \& H.C.Lima & Carvoeiro & 1 & 7 & \\
\hline & Vachellia farnesiana (L.) Wight \& Arn. & Aromita & 2 & $1,3,6,11$ & 51745 - ESA \\
\hline & Vatairea macrocarpa (Benth.) Ducke & Angelim & 1 & 4,13 & \\
\hline Icacinaceae & Emmotum nitens (Benth.) Miers & Faia & 1 & $5,6,10,13$ & \\
\hline \multirow[t]{4}{*}{ Lamiaceae } & Aegiphila integrifolia (Jacq.) Moldenke & & 1 & 1,4 & 51763 - ESA \\
\hline & A. verticillata Vell. & Milho-de-grilo & 1 & 13 & \\
\hline & A. vitelliniflora Walp. & & 2 & $1,4,5,6,9,10,14,15$ & 51764 - ESA \\
\hline & Vitex cymosa Bertero ex Spreng. & Tarumã & 1 & $\begin{array}{c}1,4,5,6,9,10,11,12,13 \\
14,15\end{array}$ & $\begin{array}{l}51765 \text { - ESA; } \\
17345 \text { - CGMS }\end{array}$ \\
\hline \multirow[t]{13}{*}{ Lauraceae } & Endlicheria paniculata (Spreng.) J.F.Macbr. & Canela-do-brejo & 1 & 4 & \\
\hline & Licaria triandra (Sw.) Kosterm. & Canela & 1 & 1 & 51766 - ESA \\
\hline & Nectandra cissiflora Ness & Canela & 1 & $6,10,13$ & $\begin{array}{l}\text { 17447, 17449, } \\
17448 \text { - CGMS }\end{array}$ \\
\hline & N. hihua (Ruiz \& Pav.) Rohwer & Canela & 1 & $1,5,8,9,10,13$ & $\begin{array}{l}51767 \text { - ESA; } \\
17347 \text { - CGMS }\end{array}$ \\
\hline & N. lanceolata Ness & Canela-amarela & 1 & 6,11 & \\
\hline & N. megapotamica (Spreng.) Mez & Canela-fedida & 1 & $1,4,12,14,15$ & 51768 - ESA \\
\hline & N. membranacea (Sw.) Griseb. & Canela-branca & 1 & $2,5,9,10,12,13,14,15$ & 12943 - CGMS \\
\hline & Ocotea corymbosa (Meisn.) Nees & Canelinha & 1 & 12,14 & \\
\hline & O. diospyrifolia (Meisn.) Mez & $\begin{array}{l}\text { Caneleira, canela- } \\
\text { branca }\end{array}$ & 1 & 6,11 & \\
\hline & O. minarum (Nees \& Mart.) Mez & Canela-vassoura & 1 & $4,5,6,7,10,13,15$ & \\
\hline & O. puberula (Rich.) Nees & Canela-guaicá & 1 & $5,6,7,10,13,15$ & \\
\hline & O. velloziana (Meissn.) Mez & Canela-branca & 1 & $3,5,10,12,14,15$ & \\
\hline & O. velutina (Nees) Rohwer & Canelão-amarelo & 1 & 6,11 & \\
\hline Lecythidaceae & Cariniana estrellensis (Raddi) Kuntze & Jequitibá-branco & 1 & $6,14,15$ & \\
\hline Loganiaceae & Strychnos pseudoquina A. St.Hil. & Quina-do-cerrado & 1 & $\begin{array}{c}4,5,7,8,9,10,11,13 \\
14,15\end{array}$ & \\
\hline \multirow[t]{2}{*}{ Lythraceae } & Adenaria floribunda Kunth & Veludo & 2 & $4,5,6,8,10,11,13,15$ & \\
\hline & Lafoensia pacari A. St.Hil. & Mangabeira-brava & 1 & $4,5,6,7,8,10,13,15$ & \\
\hline \multirow[t]{11}{*}{ Malpighiaceae } & Bunchosia paraguariensis Nied. & & 1 & $1,6,11$ & 51774 - ESA \\
\hline & Byrsonima coccolobifolia Kunth & Sumanera & 1 & $4,5,6,7,8,10,13$ & 17451 - CGMS \\
\hline & B. crassifolia (L.) Kunth & Canjicão & 1 & $4,5,6,8,10,13$ & \\
\hline & B. cydoniifolia A.Juss. & Canjiqueira & 2 & 4,11 & $\begin{array}{c}17453,17455- \\
\text { CGMS }\end{array}$ \\
\hline & B. pachyphylla A. Juss. & Murici & 1 & 7 & \\
\hline & $B$. verbascifolia $(L) D C$ & Murici-do-cerrado & 2 & $5,6,7,10,11,13,15$ & 17452 - CGMS \\
\hline & Heteropterys tomentosa A.Juss. & Nó-de-cachorro & 2 & $5,7,8,10,13,15$ & \\
\hline & H. byrsonimiifolia A.Juss. & Murici-macho & 1 & 4,7 & 17452 - CGMS \\
\hline & H. hypericifolia A. Juss. & & 2 & $1,5,7,10,13$ & 51775 - ESA \\
\hline & Peixotoa cordistipula A. Juss. & & 2 & $5,7,10$ & \\
\hline & Thryallis laburnum S. Moore & & 1 & 1 & 51777 - ESA \\
\hline \multirow[t]{5}{*}{ Malvaceae } & Apeiba tibourbou Aubl. & Pente-de-macaco & 1 & $1,4,5,6,9,10,11,14$ & $\begin{array}{l}51778 \text { - ESA; } \\
17456 \text { - CGMS }\end{array}$ \\
\hline & $\begin{array}{l}\text { Bastardiopsis densiflora (Hook. \& Arn.) } \\
\text { Hassl. }\end{array}$ & $\begin{array}{l}\text { Louro-branco, } \\
\text { vassourão }\end{array}$ & 1 & $1,2,4,5,6,10,12,14,15$ & $\begin{array}{l}51779 \text { - ESA; } \\
12942 \text { - CGMS }\end{array}$ \\
\hline & Ceiba boliviana Britten \& Baker $f$. & Barriguda & 1 & 15 & \\
\hline & C. pubiflora (A.St.-Hil.) K.Schum. & $\begin{array}{l}\text { Barriguda-do- } \\
\text { pantanal }\end{array}$ & 1 & 6,11 & \\
\hline & C. speciosa (A.St.-Hil.) Ravenna & Paineira-rosa & 1 & $5,6,10,11,13,14,15$ & \\
\hline
\end{tabular}


Quadro 2. Cont.

\begin{tabular}{|c|c|c|c|c|c|}
\hline Família & Espécie & Nome popular & $\begin{array}{l}\text { Forma } \\
\text { biológica }\end{array}$ & Fonte & $\begin{array}{l}\text { Referência em } \\
\text { Herbário }\end{array}$ \\
\hline & Eriotheca gracilipes (K.Schum.) A.Robyns & Paina & 1 & $6,8,10,11,13,14,15$ & \\
\hline & E. pubescens (Mart. \& Zucc.) Schott \& Endl & Paineira-do-cerrado & 1 & $4,7,13$ & \\
\hline & Guazuma ulmifolia Lam. & Chico-magro & 1 & $\begin{array}{c}1,2,4,5,6,8,9,10,11 \\
12,13,14,15\end{array}$ & $\begin{array}{l}\text { 51781, } 51782 \\
\text { - ESA; } 17351 \text {, } \\
12962 \text { - CGMS }\end{array}$ \\
\hline & Helicteres guazumaefolia H.B.K. & Rosquinha & 2 & 8,10 & \\
\hline & H. lhotzkyana (Schott \& Endl.) K.Schum. & Sacarrolha & 1 & $\begin{array}{c}1,4,5,6,7,8,9,10,11 \\
12,13,14,15\end{array}$ & $\begin{array}{l}51783 \text { - ESA; } \\
17353 \text { - CGMS }\end{array}$ \\
\hline & Luehea candicans Mart. \& Zucc & Açoita-cavalo & 1 & $\begin{array}{c}4,5,6,7,8,9,10,11 \\
12,14\end{array}$ & \\
\hline & L. divaricata Mart. & Açoita-cavalo-miúdo & 1 & $5,6,7,9,10,11,13,14$ & \\
\hline & L. grandiflora Mart. \& Zucc. & Açoita-cavalo & 1 & $\begin{array}{c}4,5,6,7,8,9,10,11,12 \\
13,14,15\end{array}$ & \\
\hline & L. paniculata Mart. & Açoita-cavalo & 1 & $4,5,6,7,8,10,11,14,15$ & \\
\hline & Melochia parvifolia Kunth & Malvavinha & 2 & $5,6,8,9,10,11,13$ & \\
\hline & Pavonia grandiflora A.St.-Hil. & Malvão & 2 & 7 & \\
\hline & $\begin{array}{l}\text { Pseudobombax grandiflorum (Cav.) } \\
\text { A.Robyns }\end{array}$ & Embiruçu & 1 & $4,6,11,12,14$ & \\
\hline & P. longiflorum (Mart. \& Zucc.) A.Robyns & Mamonarana & 1 & $4,5,9,10,13$ & \\
\hline & P. tomentosum (Mart. \& Zucc.) A.Robyns & Embiruçu & 1 & $\begin{array}{c}4,5,6,7,8,9,10,11,12 \\
13,14,15\end{array}$ & \\
\hline & Sterculia apetala (Jacq.) H. Karst. & Mandovi & 1 & $1,4,6,11,14,15$ & 51785 - ESA \\
\hline & S. striata A.St.-Hil. \& Naudin & Mandovi & 1 & $5,6,8,10,11,13$ & \\
\hline \multirow[t]{2}{*}{ Marcgraviaceae } & Norantea guianensis Aublet. & Flor-de-papagaio & 2 & 4 & 17457 - CGMS \\
\hline & $\begin{array}{l}\text { Schwartzia adamantium (Cambess.) } \\
\text { Bedell ex Gir.-Cañas }\end{array}$ & Mel-de-arara & 2 & 4 & \\
\hline \multirow[t]{7}{*}{ Melastomataceae } & Clidemia bullosa DC. & & 2 & $5,6,9,10,11,13,14,15$ & \\
\hline & Miconia albicans (Sw.) Triana & Folha-branca & 2 & $\begin{array}{c}4,5,6,8,9,10,11,13 \\
14,15\end{array}$ & \\
\hline & M. burchellii Triana & Pixirica & 1 & 4,13 & \\
\hline & M. discolor DC. & Pixirica & 1 & 14 & \\
\hline & M. ferruginata (Aubl.) Cogn. & Pixirica & 2 & 4,13 & 17458 - CGMS \\
\hline & M. prasina $(S w) D C$. & & 2 & 13 & \\
\hline & M. pusilliflora (DC.) Naudin & & 1 & 14 & \\
\hline \multirow[t]{3}{*}{ Meliaceae } & Cabralea canjerana (Vell.) Mart. & Canjarana & 1 & $1,5,6,10,12,13,14,15$ & 51786 - ESA \\
\hline & Cedrela fissilis Vell. & $\begin{array}{l}\text { Cedro-branco, cedro- } \\
\text { rosa }\end{array}$ & 1 & $\begin{array}{c}1,2,4,5,6,9,10,11,12 \\
13,14,15\end{array}$ & 51787 - ESA \\
\hline & C. odorata $L$. & Cedro-d'água & 1 & 5,10 & \\
\hline \multirow[t]{8}{*}{ Mekiaceae } & Guarea guidonia (L.) Sleumer & $\begin{array}{l}\text { Canjambo, } \\
\text { marinheiro }\end{array}$ & 1 & $\begin{array}{c}1,2,4,5,6,8,9,10,12 \\
13,14,15\end{array}$ & $\begin{array}{c}51789 \text { - ESA; } \\
\text { 17359, } 12957 \text { - } \\
\text { CGMS }\end{array}$ \\
\hline & G. kuntiana A. Juss & $\begin{array}{l}\text { Canjambo, } \\
\text { marinheiro }\end{array}$ & 1 & $\begin{array}{c}1,2,4,5,6,8,9,10,12 \\
13,14\end{array}$ & $\begin{array}{c}51788 \text { - ESA; } \\
\text { 17354, } 12955- \\
\text { CGMS }\end{array}$ \\
\hline & Trichilia casaretti C. DC. & Catiguá-branco & 1 & $1,12,14$ & $\begin{array}{l}51790 \text { - ESA; } \\
17360 \text { - CGMS }\end{array}$ \\
\hline & T. catigua A. Juss. & Pombeiro & 1 & $1,6,11$ & 51791 - ESA \\
\hline & T. claussenii C. DC. & Catiguá -vermelho & 1 & $\begin{array}{c}1,2,5,6,8,9,10,12,13 \\
14,15\end{array}$ & $\begin{array}{l}51792 \text { - ESA; } \\
12956 \text { - CGMS }\end{array}$ \\
\hline & T. elegans A. Juss. & Catiguá-ervilha & 1 & 1,3 & 51793 - ESA \\
\hline & T. hirta $L$. & Cachuá & 1 & $\begin{array}{c}1,2,4,6,8,10,11,12 \\
14,15\end{array}$ & $\begin{array}{c}51794 \text { - ESA; } \\
\text { 17361, } 12953- \\
\text { CGMS }\end{array}$ \\
\hline & T. pallida $S w$. & Catiguá & 1 & $1,2,5,9,10,13$ & $\begin{array}{l}51795-\text { ESA; } \\
12954 \text { - CGMS }\end{array}$ \\
\hline
\end{tabular}


Quadro 2. Cont.

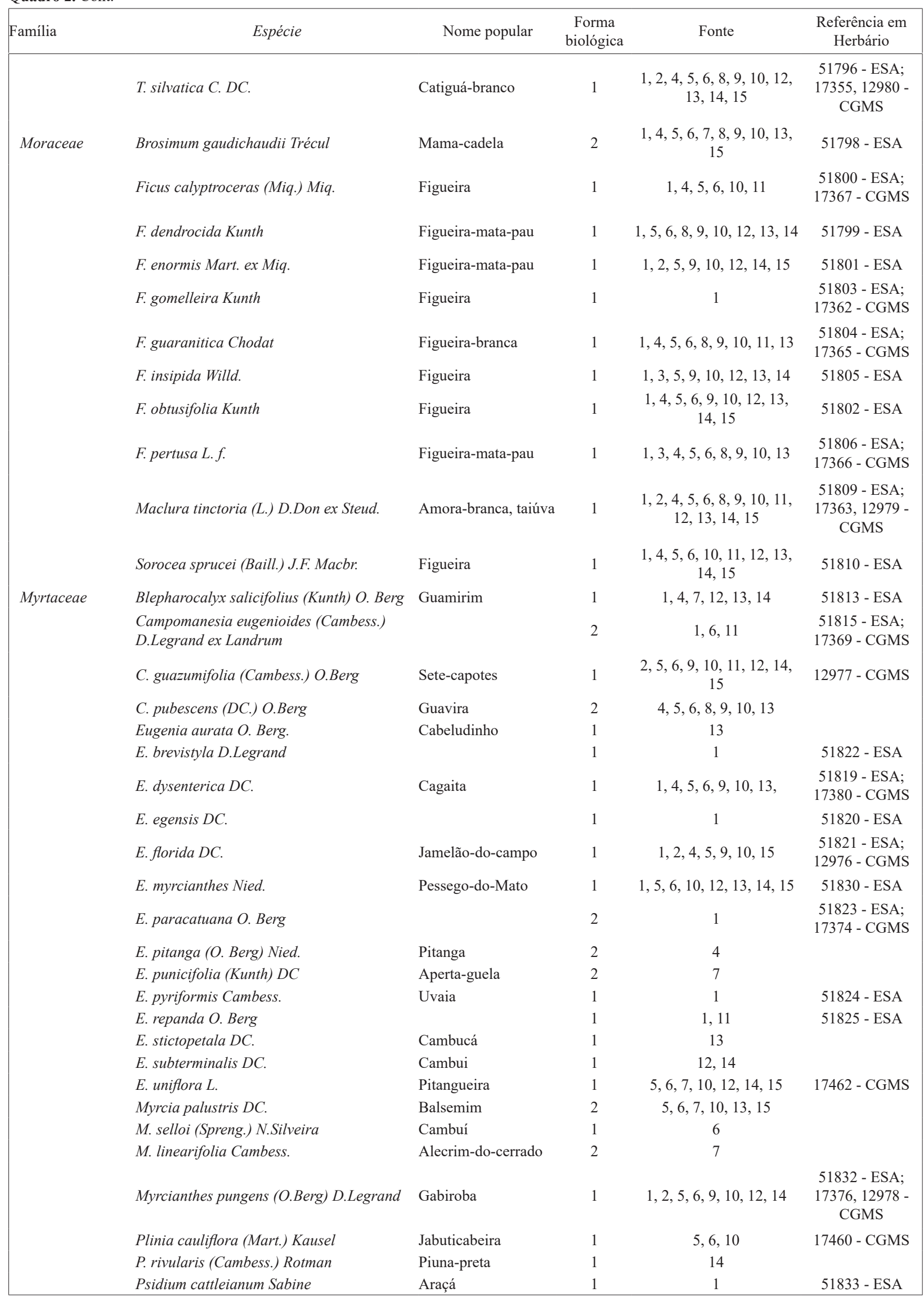


Quadro 2. Cont.

\begin{tabular}{|c|c|c|c|c|c|}
\hline Família & Espécie & Nome popular & $\begin{array}{c}\text { Forma } \\
\text { biológica }\end{array}$ & Fonte & $\begin{array}{c}\text { Referência em } \\
\text { Herbário }\end{array}$ \\
\hline & P. guajava $L$. & Goiaba & 2 & $\begin{array}{c}4,5,6,7,8,9,10,11,12 \\
13,14,15\end{array}$ & \\
\hline \multirow[t]{4}{*}{ Myrtaceae } & P. guineense $S w$. & Araçá & 2 & $\begin{array}{c}5,6,8,9,10,11,12,13 \\
14,15\end{array}$ & \\
\hline & P. myrtoides O.Berg & Araçá & 1 & $4,7,13$ & \\
\hline & P. riparium Mart. ex DC. & & 1 & 11 & \\
\hline & P. sartorianum (Nied.) Berg & Goiabinha & 1 & $\begin{array}{c}5,6,8,9,10,11,12,13 \\
14,15\end{array}$ & \\
\hline \multirow[t]{10}{*}{ Nyctaginaceae } & Bougainvillea glabra Choisy & $\begin{array}{l}\text { Três-marias, } \\
\text { primavera }\end{array}$ & 1 & & 17463 - CGMS \\
\hline & Guapira areolata (Heimerl) Lundell & & 1 & 1 & 51834 - ESA \\
\hline & $\begin{array}{l}\text { G. graciliflora (Mart. ex J. A. Schmidt) } \\
\text { Lundel }\end{array}$ & João-mole & 1 & $4,5,7,10$ & \\
\hline & G. noxia (Netto) Lundell & Caparrosa & 1 & 13 & \\
\hline & G. opposita (Vell.) Reitz & Maria-mole & 1 & $1,2,4,5,6,10,11,15$ & $\begin{array}{c}51835 \text { - ESA; } \\
\text { 17383, } 12975 \text { - } \\
\text { CGMS }\end{array}$ \\
\hline & Neea hermaphrodita S. Moore & & 1 & $1,5,6,10,11$ & 51839 - ESA \\
\hline & N. theifera Oerst. & Caparrosa & 1 & 13 & \\
\hline & Pisonia aculeata L. & & 1 & 1 & 51836 - ESA \\
\hline & P. ambigua Heimerl & Carrapicho & 1 & 15 & \\
\hline & P. zapallo Griseb. & Carrapicho & 2 & $1,4,5,6,9,10,11$ & 51837 - ESA \\
\hline Olacaceae & Ximenia americana $L$. & Limãozinho & 1 & $1,2,5,6,8,9,10,11,15$ & $\begin{array}{l}51840 \text { - ESA; } \\
12970 \text { - CGMS }\end{array}$ \\
\hline \multirow[t]{2}{*}{ Oleaceae } & Chionanthus filiformis (Vell.) P.S. Green & & 1 & 1 & $\begin{array}{l}51841 \text { - ESA; } \\
17384 \text { - CGMS }\end{array}$ \\
\hline & $\begin{array}{l}\text { Priogymnanthus hasslerianus (Chodat) P.S. } \\
\text { Green }\end{array}$ & Osso-de-burro & 1 & $1,4,5,6,8,9,10,11,15$ & $\begin{array}{l}51842 \text { - ESA; } \\
17385 \text { - CGMS }\end{array}$ \\
\hline Opiliaceae & $\begin{array}{l}\text { Agonandra brasiliensis Miers ex Benth. \& } \\
\text { Hook. } f .\end{array}$ & $\begin{array}{l}\text { Tingue-cuia, cerveja- } \\
\text { de-pobre }\end{array}$ & 1 & $1,2,4,5,6,9,10,13$ & $\begin{array}{l}51843 \text { - ESA; } \\
12972 \text { - CGMS }\end{array}$ \\
\hline \multirow[t]{2}{*}{ Phyllanthaceae } & Phyllanthus chacoensis Morong & Jacarepito & 1 & 6,11 & \\
\hline & P. sellowianus (Klotzsch) Müll. Arg. & Sarandi-branco & 2 & 1 & 51705 - ESA \\
\hline \multirow[t]{4}{*}{ Phyllanthaceae } & Margaritaria nobilis L. $f$. & Figueirinha & 1 & $1,5,6,9,10$ & $\begin{array}{l}51848 \text { - ESA; } \\
17386 \text { - CGMS }\end{array}$ \\
\hline & Gallesia integrifolia (Spreng.) Harms & Pau-alho & 1 & $1,5,6,10,11,13,15$ & 51858 - ESA \\
\hline & Rivina humilis $L$. & & 2 & $\begin{array}{c}1,6,9,10,11,12,13 \\
14,15\end{array}$ & 51859 - ESA \\
\hline & Phytolacca dioica $L$. & Cebolão & 1 & $5,6,10$ & \\
\hline \multirow[t]{5}{*}{ Piperaceae } & Piper aduncum L. & $\begin{array}{l}\text { Falso-jaborandi, } \\
\text { pimenta-do-mato }\end{array}$ & 2 & $\begin{array}{l}1,2,3,4,5,6,9,10,11 \\
12,13,14,15\end{array}$ & $\begin{array}{c}51849,51851 \\
\text { - ESA; } 12959 \text { - } \\
\text { CGMS }\end{array}$ \\
\hline & $P$. amalago $L$. & & 2 & $1,6,11$ & 51850 - ESA \\
\hline & P. arboreum Aubl. & Falso-jaborandi & 2 & $1,12,14$ & 51852 - ESA \\
\hline & P. chimonanthifolium Kunth & & 2 & 1 & 51853 - ESA \\
\hline & P. tuberculatum Jacq. & Pimenta-de-macaco & 2 & $\begin{array}{c}1,3,4,5,6,9,10,11,12 \\
13,14,15\end{array}$ & $\begin{array}{l}51855-\text { ESA; } \\
\text { 17464, } 12973- \\
\text { CGMS }\end{array}$ \\
\hline \multirow[t]{7}{*}{ Polygonaceae } & Coccoloba cujabensis Wedd. & Canjiquinha & 2 & $1,5,6,10,11,13$ & 51862 - ESA \\
\hline & C. mollis Casar & Canjiquinha & 1 & 4,6 & 17465 - CGMS \\
\hline & C. paraguariensis Lindau & Canjiquinha & 1 & 11 & \\
\hline & C. parimensis Benth. & Canjiquinha & 1 & 4 & \\
\hline & C. rigida Meisn. & Canjiquinha & 2 & $5,10,11,13$ & \\
\hline & Ruprechtia exploratricis Sandwith & Pele-de-velho & 1 & 6,11 & \\
\hline & R. triflora Griseb. & & 1 & 11 & \\
\hline
\end{tabular}


Quadro 2. Cont.

\begin{tabular}{|c|c|c|c|c|c|}
\hline Família & Espécie & Nome popular & $\begin{array}{c}\text { Forma } \\
\text { biológica }\end{array}$ & Fonte & $\begin{array}{l}\text { Referência em } \\
\text { Herbário }\end{array}$ \\
\hline & Triplaris americana $L$. & Novateiro & 1 & $1,4,6$ & $\begin{array}{c}51861 \text { - ESA; } \\
\text { 17389, } 17466- \\
\text { CGMS }\end{array}$ \\
\hline \multirow[t]{4}{*}{ Primulaceae } & Clavija nutans (Vell.) B. Ståhl & Chá-de-bugre & 2 & $1,3,5,6,8,9,10,11,15$ & 51916 - ESA \\
\hline & $\begin{array}{l}\text { Myrsine coriacea (Sw.) R.Br. ex Roem. \& } \\
\text { Schult. }\end{array}$ & Capororoca & 1 & $\begin{array}{c}1,4,5,6,7,8,10,11,12 \\
13,14,15\end{array}$ & 51811 - ESA \\
\hline & M. guianensis (Aubl.) Kuntze & Capororoca & 1 & $\begin{array}{c}1,3,4,5,6,7,8,9,10,11 \\
12,13,14,15\end{array}$ & 51812 - ESA \\
\hline & M. umbellata Mart. & Capororocão & 1 & $2,5,10$ & \\
\hline \multirow[t]{2}{*}{ Proteaceae } & $\begin{array}{l}\text { Roupala montana var. brasiliensis (Klotzsch, } \\
\text { K.S.Edwards }\end{array}$ & ${ }^{2)}$ Carvalho-brasileiro & 1 & $5,10,12,14$ & 17467 - CGMS \\
\hline & R. montana Aubl. var. montana & Carne-de-vaca & 1 & $4,5,10,13$ & \\
\hline \multirow[t]{3}{*}{ Rhamnaceae } & Colubrina glandulosa Perkins & & 1 & $1,5,6,10,11$ & 51863 - ESA \\
\hline & Rhamnidium elaeocarpum Reissek & Cabriteiro & 1 & $\begin{array}{c}1,2,3,4,5,6,8,9,10,11 \\
13,14,15\end{array}$ & $\begin{array}{c}51866 \text { - ESA; } \\
\text { 12969, } 17390- \\
\text { CGMS }\end{array}$ \\
\hline & Ziziphus oblongifolia S. Moore & Olho-de-boi & 1 & 6,11 & \\
\hline \multirow[t]{15}{*}{ Rubiaceae } & Alibertia edulis (Rich.) A.Rich. & Marmelo & 1 & $\begin{array}{c}1,3,4,5,6,7,8,9,10,12 \\
14,15\end{array}$ & $\begin{array}{c}51868 \text { - ESA; } \\
\text { 17470, } 17472- \\
\text { CGMS }\end{array}$ \\
\hline & Calycophyllum multiflorum Griseb. & Castelo & 1 & $\begin{array}{c}1,5,6,9,10,11,12,14 \\
15\end{array}$ & $\begin{array}{l}51870 \text { - ESA; } \\
17396 \text { - CGMS }\end{array}$ \\
\hline & Cephalanthus glabratus (Spreng.) K.Schum. & Sarã & 2 & 10 & \\
\hline & Chomelia obtusa Cham. \& Schltdl. & $\begin{array}{l}\text { Espinheiro-do- } \\
\text { cerrado }\end{array}$ & 2 & $5,6,8,9,10,11,15$ & \\
\hline & C. pohliana Müll. Arg. & Espinheiro-do-mato & 1 & $3,12,14$ & \\
\hline & Cordiera sessilis (Vell.) Kuntze & Marmelo & 1 & $1,2,4,5,6,7,8,9,10,13$ & $\begin{array}{l}51867 \text { - ESA; } \\
12968 \text { - CGMS }\end{array}$ \\
\hline & Coussarea hydrangeifolia (Benth.) Müll.Arg. & Falsa-quina & 1 & 10 & \\
\hline & Ferdinandusa elliptica (Pohl) Pohl & Brinco-d'água & 1 & 4,13 & \\
\hline & Genipa americana $L$. & Jenipapo & 1 & $\begin{array}{c}1,4,5,6,7,9,10,11,12 \\
13,14,15\end{array}$ & $\begin{array}{c}51872 \text { - ESA; } \\
\text { 17394, } 17473- \\
\text { CGMS }\end{array}$ \\
\hline & Guettarda viburnoides Cham. \& Schltdl. & Veludo & 1 & $1,4,5,6,9,10,13$ & $\begin{array}{c}51873 \text { - ESA; } \\
\text { 17393, } 17471- \\
\text { CGMS }\end{array}$ \\
\hline & Psychotria carthagenensis Jacq. & Pasto-de-anta & 2 & $\begin{array}{c}1,2,3,4,5,6,8,9,10,11 \\
12,13,14,15\end{array}$ & $\begin{array}{c}51874-\text { ESA; } \\
\text { 17392, } 12967- \\
\text { CGMS }\end{array}$ \\
\hline & Randia armata (Sw.) DC. & Veludo-de-espinho & 2 & $\begin{array}{c}1,3,4,5,6,8,9,10,11 \\
12,13,14,15\end{array}$ & 51876 - ESA \\
\hline & Rudgea viburnoides (Cham.) Benth. & Congonha & 1 & $3,5,8,10,13$ & \\
\hline & Sphinctanthus hasslerianus Chodat & Rebenta-laço & 2 & $5,6,8,10,11,13,15$ & \\
\hline & $\begin{array}{l}\text { Tocoyena formosa (Cham. \& Schltdl.) } \\
\text { K.Schum. }\end{array}$ & Olho-de-boi & 2 & $4,5,6,7,8,10,13,14$ & 17474 - CGMS \\
\hline \multirow[t]{7}{*}{ Rutaceae } & Balfourodendron riedelianum (Engl.) Engl. & Pau-marfim & 1 & $1,4,6,11,12,14,15$ & $\begin{array}{l}51877 \text { - ESA; } \\
17402 \text { - CGMS }\end{array}$ \\
\hline & Esenbeckia leiocarpa Engl. & Guarantã & 1 & $1,5,6,10,11,12,14,15$ & 51879 - ESA \\
\hline & Helietta apiculata Benth. & $\begin{array}{l}\text { Cun-cun, canela-de- } \\
\text { cutia }\end{array}$ & 1 & $\begin{array}{c}1,2,5,6,8,9,10,11,12 \\
13,14,15\end{array}$ & $\begin{array}{c}51881 \text { - ESA; } \\
\text { 17411, } 12974- \\
\text { CGMS }\end{array}$ \\
\hline & Pilocarpus pauciflorus A.St.-Hil. & Jaborandi & 1 & 14 & \\
\hline & P. pennatifolius Lem. & Jaborandi & 2 & $1,4,12,14$ & 51880 - ESA \\
\hline & Zanthoxylum caribaeum Lam. & Laranjeira-brava & 1 & $5,6,10,11,12,13,14,15$ & \\
\hline & Z. fagara (L.) Sarg. & Mamica-de-porca & 1 & $8,12,14$ & \\
\hline
\end{tabular}


Quadro 2. Cont.

\begin{tabular}{|c|c|c|c|c|c|}
\hline Família & Espécie & Nome popular & $\begin{array}{c}\text { Forma } \\
\text { biológica }\end{array}$ & Fonte & $\begin{array}{l}\text { Referência em } \\
\text { Herbário }\end{array}$ \\
\hline \multirow{7}{*}{ Salicaceae } & $\begin{array}{l}\text { Z. rigidum subsp. hasslerianum (Chodat) } \\
\text { Reynel }\end{array}$ & Mamica-de-porca & 1 & $\begin{array}{c}1,2,5,6,8,9,10,12,13 \\
14,15\end{array}$ & $\begin{array}{c}51882 \text { - ESA; } \\
\text { 17399, } 12981 \text { - } \\
\text { CGMS }\end{array}$ \\
\hline & Z. rhoifolium Lam. & Mamica-de-porca & 1 & $\begin{array}{c}1,4,5,6,7,8,9,10,11 \\
12,13,14,15\end{array}$ & 51883 - ESA \\
\hline & Banara arguta Briq. & Sardinheira & 1 & $5,6,10,11,13$ & \\
\hline & Casearia decandra Jacq. & Guaçatunga & 1 & $\begin{array}{c}2,4,5,6,7,9,10,11,12 \\
13,14,15\end{array}$ & 12945 - CGMS \\
\hline & C. gossypiosperma Briq. & Espeteiro & 1 & $\begin{array}{c}1,4,5,6,8,9,10,11,12 \\
13,14,15\end{array}$ & $\begin{array}{l}51887 \text { - ESA; } \\
17409 \text { - CGMS }\end{array}$ \\
\hline & C. mariquitensis Kunth & & 1 & $5,10,13$ & \\
\hline & C. rupestris Eichler & Pururuca & 1 & $\begin{array}{c}1,4,5,6,8,9,10,11,12 \\
13,14,15\end{array}$ & $\begin{array}{l}51888 \text { - ESA; } \\
17403 \text { - CGMS }\end{array}$ \\
\hline \multirow{9}{*}{ Sapindaceae } & C. sylvestris $S w$. & Guaçatonga & 1 & $\begin{array}{l}1,2,3,4,5,6,7,8,9,10 \\
\quad 11,12,13,14,15\end{array}$ & $\begin{array}{c}51890 \text { - ESA; } \\
17406,17475 \\
12946 \text { - CGMS }\end{array}$ \\
\hline & Prockia crucis P. Browne ex $L$. & & 2 & $\begin{array}{c}1,4,5,6,8,9,10,11,12 \\
13,14,15\end{array}$ & 51891 - ESA \\
\hline & Salix humboldtiana Willd. & Sarã & 1 & 6 & \\
\hline & Xylosma venosa N.E. Br. & Sucará, espinheiro & 1 & $1,6,11$ & 51892 - ESA \\
\hline & $\begin{array}{l}\text { Allophylus edulis (A.St.-Hil. et al.) } \\
\text { Hieron. ex Niederl. }\end{array}$ & Vacum & 1 & $\begin{array}{c}1,2,5,6,8,9,10,11,12 \\
14,15\end{array}$ & $\begin{array}{c}51893 \text { - ESA; } \\
\text { 17413, } 12964 \text { - } \\
\text { CGMS }\end{array}$ \\
\hline & Averrhoidium paraguaiense Radlk. & Maria-Preta & 1 & $\begin{array}{c}1,2,4,5,6,8,9,10,11 \\
12,14,15\end{array}$ & $\begin{array}{c}51894 \text { - ESA; } \\
\text { 17412, } 12966 \text { - } \\
\text { CGMS }\end{array}$ \\
\hline & Cupania castaneaefolia Mart. & Camboatã & 1 & $\begin{array}{c}1,2,4,5,6,9,10,11,12 \\
13,14,15\end{array}$ & $\begin{array}{l}51896 \text { - ESA; } \\
17410 \text { - CGMS }\end{array}$ \\
\hline & Cupania vernalis Cambess. & Camboatá & 1 & $5,6,10,11,12,13,14,15$ & \\
\hline & Diatenopteryx sorbifolia Radlk. & Maria-preta & 1 & $\begin{array}{c}1,5,6,8,9,10,11,12,13 \\
14,15\end{array}$ & 51897 - ESA \\
\hline \multirow{16}{*}{ Sapotaceae } & Dilodendron bipinnatum Radlk. & Maria-mole & 1 & $\begin{array}{c}1,4,5,6,8,9,10,11,12 \\
13,14,15\end{array}$ & $\begin{array}{l}51898 \text { - ESA; } \\
17414 \text { - CGMS }\end{array}$ \\
\hline & Diplokeleba floribunda N.E.Br. & Canela-de-cutia & 1 & 6,11 & \\
\hline & Magonia pubescens A. St.Hil. & Timbó & 1 & $\begin{array}{c}1,4,5,6,7,8,10,13 \\
14,15\end{array}$ & 51899 - ESA \\
\hline & Matayba elaeagnoides Radlk. & Camboatá & 1 & $12,13,14$ & \\
\hline & M. guianensis Aubl. & Camboatá & 2 & 4,7 & \\
\hline & Melicoccus lepidopetalus Radlk. & Água-pombo-macho & 1 & 6,11 & 17476 - CGMS \\
\hline & Sapindus saponaria L. & Saboneteira & 1 & 1,4 & 51901 - ESA \\
\hline & Talisia esculenta (Cambess.) Radlk. & Pitomba & 1 & $\begin{array}{c}1,2,4,5,6,8,9,10,11 \\
15\end{array}$ & $\begin{array}{l}51906 \text { - ESA; } \\
12965 \text { - CGMS }\end{array}$ \\
\hline & Toulicia tomentosa Radlk. & Balãozinho & 2 & 9 & \\
\hline & $\begin{array}{l}\text { Chrysophyllum gonocarpum (Mart. \& } \\
\text { Eichler ex Miq.) Engl. }\end{array}$ & Aguaí & 1 & $\begin{array}{c}1,2,4,5,6,9,10,11,12 \\
14,15\end{array}$ & $\begin{array}{l}51907 \text { - ESA; } \\
12963 \text { - CGMS }\end{array}$ \\
\hline & C. marginatum (Hook. \& Arn.) Radlk. & Leiteiro & 1 & $1,4,6,11$ & $\begin{array}{c}51908 \text { - ESA; } \\
\text { 17418, } 17477 \text { - } \\
\text { CGMS }\end{array}$ \\
\hline & Pouteria gardnerii (Mart. \& Miq.) Baehni & Leiteiro-folha-miuda & 1 & $5,6,8,10,11$ & \\
\hline & P. glomerata (Miq.) Radlk. & Laranjinha & 2 & $1,6,11$ & 51909 - ESA \\
\hline & P. ramiflora (Mart.) Radlk. & $\begin{array}{l}\text { Curiola, fruta-de- } \\
\text { veado }\end{array}$ & 1 & $4,5,6,7,10,13$ & \\
\hline & P. torta (Mart.) Radlk. & Grão-de-galo & 1 & $4,5,7,10,13$ & 17478 - CGMS \\
\hline & $\begin{array}{l}\text { Sideroxylon obtusifolium (Roem. \& Schult.) } \\
\text { T.D.Penn. }\end{array}$ & Laranjinha & 1 & 6,11 & \\
\hline
\end{tabular}


Quadro 2. Cont.

\begin{tabular}{|c|c|c|c|c|c|}
\hline Família & Espécie & Nome popular & $\begin{array}{c}\text { Forma } \\
\text { biológica }\end{array}$ & Fonte & $\begin{array}{l}\text { Referência em } \\
\text { Herbário }\end{array}$ \\
\hline \multirow[t]{2}{*}{ Simaroubaceae } & Castela tweedii Planch. & & 2 & $1,5,10$ & $\begin{array}{l}\text { 51910- ESA; } \\
17419 \text { - CGMS }\end{array}$ \\
\hline & Simarouba versicolor A. St.-Hill. & Mata-cachorro, perdiz & 1 & $4,5,6,7,10,11,13,15$ & 17479 - CGMS \\
\hline \multirow[t]{7}{*}{ Solanaceae } & Cestrum mariquitense Kunth & Coerana & 2 & $5,7,9,10,12,14,15$ & \\
\hline & C. strigilatum Ruiz \& Pav. & Coerana, pau-de-rato & 2 & $\begin{array}{c}1,4,5,6,8,9,10,11,12 \\
14,15\end{array}$ & $\begin{array}{c}51780,51912- \\
\text { ESA }\end{array}$ \\
\hline & Solanum bonariense $L$. & Jurubeba-brava & 2 & $5,6,9,10,11,13,15$ & \\
\hline & S. glaucophyllum Desf. & Espichadeira & 2 & $10,11,12,13$ & \\
\hline & S. lycocarpum A. St.-Hill. & Lobeira & 2 & $4,5,6,7,10,13$ & 17480 - CGMS \\
\hline & S. paniculatum L. & Jurubeba & 2 & $6,11,14,15$ & \\
\hline & S. stipulatum Vell. & Fumo-bravo & 1 & $5,8,9,10,13$ & \\
\hline \multirow[t]{3}{*}{ Styracaceae } & Styrax camporum Pohl & Benjoeiro & 1 & $4,5,6,10,11,13$ & \\
\hline & S. ferrugineus Ness \& Mart. & $\begin{array}{l}\text { Laranjinha-do- } \\
\text { cerrado }\end{array}$ & 1 & $3,4,5,6,7,10,11,13$ & \\
\hline & S. pohlii A. DC. & Benjoeiro & 1 & $\underset{15}{4,5,6,8,10,11,13,14}$ & \\
\hline \multirow[t]{4}{*}{ Urticaceae } & Boehmeria caudata $S w$. & & 2 & 1 & $\begin{array}{l}51917 \text { - ESA; } \\
17420 \text { - CGMS }\end{array}$ \\
\hline & Cecropia pachystachya Trécul & Embaúba & 1 & $\begin{array}{c}1,2,4,5,6,7,8,9,10,11 \\
12,13,14,15\end{array}$ & $\begin{array}{l}51918 \text { - ESA; } \\
12948 \text { - CGMS }\end{array}$ \\
\hline & Urera aurantiaca Wedd. & Urtiga-de-pacu & 2 & $5,6,9,10,11,12,14,15$ & \\
\hline & U. baccifera (L.) Gaudich. ex Wedd. & Urtigão & 1 & $1,4,6,11,12,14$ & $\begin{array}{l}51919 \text { - ESA; } \\
17421 \text { - CGMS }\end{array}$ \\
\hline \multirow[t]{5}{*}{ Verbenaceae } & Aloysia virgata (Ruiz \& Pav.) Juss. & Lixa & 1 & $\begin{array}{c}1,3,4,5,6,9,10,11,13 \\
14,15\end{array}$ & 51922 - ESA \\
\hline & Cytharexylum myrianthum Cham. & Pau-viola & 1 & $\begin{array}{c}1,4,5,6,9,10,11,12 \\
14,15\end{array}$ & 51923 - ESA \\
\hline & Lantana canescens Kunth & Lantana & 2 & $\begin{array}{c}1,3,4,5,6,8,9,10,11 \\
13,14,15\end{array}$ & 51924 - ESA \\
\hline & L. trifolia $L$. & Uvinha-do-campo & 2 & $1,5,6,9,10,11,13$ & 51925 - ESA \\
\hline & Lippia alba (Mill.) N.E. Br. & Cidreira-do-campo & 1 & $\underset{15}{1,5,6,9,10,11,13,14}$ & 51926 - ESA \\
\hline \multirow[t]{2}{*}{ Vochysiaceae } & Callisthene fasciculata Mart. & Carvão-branco & 1 & $4,5,6,7,8,10,13,14,15$ & 17482 - CGMS \\
\hline & C. minor Mart. & Pau-de-pilão & 1 & 8 & \\
\hline \multirow{11}{*}{$\begin{array}{l}\text { Vochysiaceae } \\
\text { (continuação) }\end{array}$} & Qualea dichotoma (Mart.) Warm. & Pau-terra & 1 & 6,13 & 17484 - CGMS \\
\hline & Q. grandiflora Mart. & Pau-terra-grande & 1 & $4,5,6,7,8,10,11,13,15$ & 17485 - CGMS \\
\hline & Q. multiflora Mart. & Pau-terra-liso & 1 & $4,5,6,7,8,10,13$ & 17481 - CGMS \\
\hline & Q. parviflora Mart. & Pau-terra-roxo & 1 & $4,5,6,7,10,13$ & 17483 - CGMS \\
\hline & Salvertia convallariodora A.St.-Hil. & Chapeu-de-couro & 1 & $4,7,13$ & \\
\hline & Vochysia divergens Pohl & Cambará & 1 & 6 & 17486 - CGMS \\
\hline & V. elliptica Mart. & Pau-doce & 1 & $4,5,10,13$ & \\
\hline & V. haenkeana Mart. & Cambarazinho & 1 & $4,13,14$ & \\
\hline & V. rufa Mart. & Pau-doce & 1 & 4 & \\
\hline & V. thyrsoidea Pohl. & Gomeira & 1 & 13 & \\
\hline & V. tucanorum Mart. & Cinzeiro & 1 & 6 & \\
\hline Zygophylaceae & Bulnesia sarmientoi Lorentz ex Griseb. & Pau-Santo & 1 & 6,11 & \\
\hline
\end{tabular}

et al. 1998). Tapirira guianensis, Guarea guidonia, Ficus insipida, Guarea kuntiana, Maclura tinctoria e Eugenia dysenterica podem ser consideradas típicas do dossel da Floresta Estacional Semidecidual Ribeirinha, enquanto que Unonopsis guatterioides e as espécies arbustivas Psychotria carthagenesis e Piper aduncum, são características do subosque nesse mesmo tipo de fisionomia florestal. As Florestas Estacionais Semideciduais Submontanas podem ser consideradas as florestas mais altas presentes no estado de Mato Grosso do Sul, tendo como árvores típicas Holocalyx balansae, Cedrela fissilis, Psidium sartorianum, Parapiptadenia rigida, 
Aspidosperma polyneuron e Guibourtia hymenaeifolia. $\mathrm{Na}$ Serra da Bodoquena foram encontradas as espécies Myracrodruon urundeuva, Ceiba pubiflora, Poincianella pluviosa var. peltophoroides, Calycophyllum multiflorum, Dilodendron bipinnatum, Peltophorum dubium e Handroanthus heptaphyllus. Árvores de ocorrência no Brasil, que também ocorrem no "Bosque Seco Chiquitano" (Vides-Almonacid et al. 2007, Jardim et al. 2003) na Bolívia, foram Capparicordis tweediana, Capparidastrum petiolare, Maytenus macrodonta, Phyllanthus chacoensis, Cnidoscolus appendiculatus, Cnidoscolus cnicodendron, Andira paniculata, Ceiba boliviana, Averrhoidium paraguiense, Leptolobium dasycarpum, Leptolobium elegans e Caesalpinia paraguariensis. As espécies Pterogyne nitens, Cecropia pachystachya, Myrsine guianensis, Trema micrantha e Guazuma ulmifolia foram espécies de distribuição ampla, pois ocorreram em várias formações florestais assim como no Cerrado.

As formações savânicas, variando de campos limpos até cerradões, compõem o bioma Cerrado em $65 \%$ do território do estado de Mato Grosso do Sul (Mato Grosso do Sul 1989). O Cerrado brasileiro é destacado, hoje, como uma das floras mais ricas do Brasil e do mundo, com mais de 12 mil espécies de plantas catalogadas (Silva et al. 2011). A diversidade de ambientes é o principal fator determinante dessa riqueza florística, que abriga frequentemente as espécies Caryocar brasiliense, Terminalia argentea, Dipteryx alata, Qualea grandiflora, Vochysia tucanorum, Callisthene fasciculata, Salvertia convallariodora, Anacardium occidentale, Hancornia speciosa, Byrsonima verbascifolia, Curatella americana, Davilla elliptica, Solanum lycocarpum, Bowdichia virgilioides, Pterodon pubescens, dentre muitas outras. Na parte sul do Pantanal, nas sub-regiões de Miranda, Nabileque e Porto Murtinho, temos a única região de Chaco (Savana Estépica) do Brasil, formada por vegetação lenhosa, baixa e espinhosa associada a campo graminoso. As árvores comuns do chaco brasileiro foram Cordia brasiliensis, Schinopsis balansae, Melicoccus lepidopetalus, Prosopis ruscifolia, Prosopis rubriflora, Bulnesia sarmientoi, Aspidosperma quebracho-blanco, Vachellia farnesiana, Capparicordis tweediana, Cynophalla retusa, Crataeva tapia, Caesalpinia paraguariensis, Phyllanthus chacoensis e Tabebuia nodosa. Também é possivel observar nesta fisionomia extensas áreas de carandazais (Copernicia alba) e paratudais (Tabebuia aurea).

Diversas espécies amostradas nas áreas estudadas encontram-se na lista das espécies ameaçadas de extinção - Red List of Threatened Plants pela International Union for Conservation of Nature and Natural Resources (IUCN 2012): Amburana cearensis, Anadenanthera colubrina var. cebil, Aspidosperma poluneuron, Myracrodron urundeuva, Attalea oleifera, Balfourodendron riedelianum, Caesalpinia paraguariensis, Cedrela fissilis, Dalbergia nigra, Dipteryx alata, Ficus calyptroceras, Ficus dendrocida e Trichilia silvatica. Com relação à lista oficial de espécies ameaçadas de extinção do Brasil (Brasil 2008), foram encontradas
Myracrodruon urundeuva, Schinopsis brasiliensis e Dalbergia nigra.

\section{AGRADECIMENTOS}

Os autores agradecem aos proprietarios das Reservas Particulares do Patrimônio Natural - RPPN, ao Instituto Chico Mendes de Conservação da Biodiversidade ICMBio, aos herbários onde o material foi depositado, aos proprietários das fazendas estudadas, à empresa MMX Mineração e Metálicos S.A, pela oportunidade de realização do Projeto Reflorestar Legal e à Fundação Neotrópica do Brasil, pela oportunidade de realização do diagnóstico do meio biótico (flora) para caracterização do Corredor de Biodiversidade Miranda-Serra da Bodoquena (Fases II e IV).

\section{REFERÊNCIAS}

Baptista-maria, V.R., Rodrigues, R.R., Damasceno-Júnior, G., Maria, F.S. \& Souza, V.C. 2009. Composição florística de florestas estacionais ribeirinhas no Estado de Mato Grosso do Sul, Brasil. Acta Botanica Brasilica. 23(2): 535-548.

Battilani, J.L., Scremin-Dias, E. \& Souza, A.L.T. 2005. Fitossociologia de um trecho da mata ciliar do rio da Prata, Jardim, MS, Brasil. Acta Botanica Brasilica 19(3): 597-608.

Brasil - Ministério do Meio Ambiente, dos Recursos Hídricos e da Amazônia Legal. 1997. Plano de Conservação da Bacia do Alto Paraguai - PCBAP, v.II,t.III. Diagnóstico dos meios físico e biótico, meio biótico. Ministério do Meio Ambiente, dos Recursos Hídricos e da Amazônia Legal - MMA/ Programa Nacional do Meio Ambiente - PNMA. Brasília. 433 p.

Brasil - Ministério do Meio Ambiente. 2008. Instrução Normativa ${ }^{\circ}$ 6, de 23 de Setembro de 2008. Disponível em: http:/www.mma.gov. br/estruturas/179/_arquivos/179_05122008033615.pdf. Acessado em 26.08.2012

Bueno, M.L., Resende, U.M. \& Gomes, T.R. 2007. Levantamento florístico nas trilhas turísticas da Reserva Particular do Patrimônio Natural São Geraldo, Bonito, Mato Grosso do Sul. Revista Brasileira de Biociências 5(2): 189-191.

Felfilli, J.M. 2003. Fragmentos florestais estacionais do Brasil central: diagnóstico e proposta de corredores ecológicos. In Fragmentação florestal e alternativas de desenvolvimento rural na região CentroOeste. (Costa, R. B. ed.). Universidade Católica Dom Bosco, Campo Grande, p.139-160.

Felfilli, J.M., Mendonça, R.C., Walter, B.M.T., Silva Junior, M.C., Nóbrega, M.G.G., Fagg, C.W., Sevilha, A.C. \& Silva, M.A. 2001. Flora Fanerogâmica das Matas de Galeria e Ciliares do Brasil Central. In: Cerrado: caracterização e recuperação de Matas de Galeria. (Ribeiro, J.F., Fonseca, C.E.L. \& Souza-Silva, J.C. eds.). Empresa Brasileira de Pesquisa Agropecuária/ Cerrados. Planaltina. p. 195-263.

International Union for Conservation of Nature - IUCN. 2012. Red List of Threatened Species. Version 2012.1. Disponível em: http://www. iucnredlist.org. Acessado em 26.08.2012.

Jardim, A., Killeen, T.J. \& Fuentes, A. 2003. Guia de arboles y arbustos del bosque seco Chiquitano, Bolivia. Fundación Amigos de la NaturalezaBolivia, Tokyo. 324 p.

Kunz, S.H., Ivanauskas, N.M., Martins S.V., Silva E. \& Stefanello D. 2009. Análise da similaridade florística entre florestas do Alto Rio Xingu, da Bacia Amazônica e do Planalto Central. Revista Brasileira de Botânica 32(4): 725-736.

Lista de Espécies da Flora do Brasil. 2012. Disponível em: http:// floradobrasil.jbrj.gov.br/2012/index. Acessado em 26.08.2012.

Mato Grosso do Sul. 1989. Macrozoneamento geoambiental de Mato Grosso do Sul. Secretaria de Estado de Planejamento do Mato Grosso do Sul. Campo Grande. 131 p. 
Mendonça, R.C., Felfilli, J.M., Silva-Junior., M.C., Rezende, A.V., Nogueira, P.E., Walter, B.M.T. \& Filgueiras, T.S. 1998. Flora vascular do cerrado. Pp. 289-539. In: Cerrado: Ambiente e Flora. (Sano, S.M.; Almeida S.P. eds.). Empresa Brasileira de Pesquisa Agropecuária/ Cerrado. Planaltina. p. 289-556.

Nunes, Y.R.F., Mendonça, A.V.R., Botezelli, L., Machado, E.L.M. \& Oliveira-Filho, A.T. 2003. Variações da fisionomia, diversidade e composição de guildas da comunidade arbórea em um fragmento de floresta semidecidual em Lavras, MG. Acta Botanica Brasilica.17 (2): 213-231.

Oliveira-Filho, A.T. \& Ratter, J.A. 1995. Study of origin of Central Brazilian forests by the analysis of plant species distribuition patterns. Edinburgh Journal of Botany 52:141-194.

Rodrigues, R.R. 2004. Uma discussão nomenclatural das formações ciliares. In: Matas ciliares: conservação e recuperação. (Rodrigues R.R., Leitão Filho H.F. eds.). Editora da Universidade de São Paulo, São Paulo, p. 91-99.
Silva, J.S.V., Pott, A., Abdon, M.M., Pott, V.J. \& Santos, K.R. 2011. Projeto Geo MS: cobertura vegetal e uso da terra do estado do Mato Grosso do Sul. Empresa Brasileira de Pesquisa Agropecuária/Informática e Agropecuária. Campinas. 64 p.

Teixeira, A.P. \& Assis, M.A. 2005. Caracterização florística e fitossociológica do componente arbustivo-arbóreo de uma floresta paludosa no Município de Rio Claro (SP), Brasil. Revista Brasileira de Botânica 28 (3): 467-476.

The Plant List. 2012. The Plant List: a working list of all plant species. Disponível em: http://www.theplantlist.org/. Acessado em 26.08.2012.

Tropicos. 2012. Tropicos. Disponível em: http://www.tropicos.org/. Acessado em 26.08.2012.

Vides-Almonacid, R., S.Reichle y F. Padilla, 2007. Planificación Ecorregional del Bosque Seco Chiquitano. Fundación para la Conservación del Bosque Chiquitano-The Nature Conservancy Santa Cruzde la Sierra. 245 p. 